\title{
Improved large-scale interstellar dust foreground model and CMB solar dipole measurement
}

\author{
J.-M. Delouis ${ }^{1}$, J.-L. Puget ${ }^{2,3}$, and L. Vibert ${ }^{2}$ \\ ${ }^{1}$ Laboratoire d'Océanographie Physique et Spatiale (LOPS), Univ. Brest, CNRS, Ifremer, IRD, Brest, France \\ e-mail: jean.marc.delouis@ifremer.fr \\ 2 Institut d'Astrophysique Spatiale, CNRS, Univ. Paris-Sud, Université Paris-Saclay, Bât. 121, 91405 Orsay Cedex, France \\ ${ }^{3}$ Ecole Normale Supérieure, Sorbonne Université, Observatoire de Paris, Université PSL, École Normale Supérieure, CNRS, Paris, \\ France
}

Received 19 February 2021 / Accepted 7 March 2021

\begin{abstract}
The cosmic microwave background (CMB) anisotropies are difficult to measure at large angular scales. In this paper, we present a new analysis of the Planck High Frequency Instrument data that brings the cosmological part and its major foreground signal close to the detector noise. The solar dipole signal induced by the motion of the Solar System with respect to the CMB is a very efficient tool for calibrating a detector or cross-calibrating sets of detectors with high accuracy. In this work, the solar dipole signal is used to extract corrections of the frequency map offsets, reducing uncertainties significantly. The solar dipole parameters are refined together with the improvement of the high-frequency foregrounds and the CMB large-scale cosmological anisotropies. The stability of the solar dipole parameters is a powerful way to control Galactic foreground removal in the component separation process. We use this stability to build a model of the spatial variations in spectral energy distribution of the interstellar dust emission. Knowledge of these variations will help future $\mathrm{CMB}$ analyses of intensity and polarization used to measure faint signals related to the optical reionization depth and the tensor-to-scalar ratio of the primordial anisotropies. The results of this work are: improved solar dipole parameters, a new interstellar dust model, and a large-scale intensity map of cosmological anisotropies.
\end{abstract}

Key words. surveys - cosmic background radiation - diffuse radiation - methods: data analysis - dust, extinction

\section{Introduction}

Accurate measurement of the diffuse extragalactic emission at microwave frequencies on the (nearly) full sky is difficult, especially at large angular scales. It requires the ability to separate the atmospheric and telescope emissions from the astronomical ones and, among those, to separate the extragalactic emission from that of the Galactic diffuse background. The cosmic microwave background (CMB) is the truly diffuse extragalactic background, and can provide key cosmological information. At the peak emission of the CMB frequencies, accurate measurements also necessitate space missions, building cryogenic telescopes and instruments, and finding compromises between constraints on the size of telescopes -meaning a limited angular resolution- and the goal to cover the whole sky.

In the last 25 years, there has been three generations of CMB space missions COBE, WMAP, and Planck, and the next one will not provide results for at least another decade. The Planck High Frequency Instrument (HFI) observed the sky (at $100,143,217,353,545$, and $857 \mathrm{GHz}$ ) with 50 bolometers cooled at $100 \mathrm{mK}$. The present and future observations from the ground use large detector arrays, but are strongly affected by the atmospheric emission above $200 \mathrm{GHz}$. Future space missions will allow us to map the CMB and the Galactic foregrounds at higher frequencies. Progress in experiments from the ground will certainly lead to a capacity to outperform Planck on the $\mathrm{CMB}$ itself up to $150 \mathrm{GHz}$ but not on the high-frequency Galactic foreground emission, which dominates the signal at higher frequencies.
The large-scale polarized $B$ modes are a major target for cosmology. Analyzing these modes requires very high sensitivity at CMB frequencies (through more detectors and better control on polarized systematic effects). It will also require better measurements of the low-frequency foreground emission with large ground-based surveys, and of high-frequency foreground emission, which is hard to measure from the ground. A key objective is therefore to exhaustively analyze the HFI data in search of information on the large-scale high-frequency foreground emission. This is the goal of the present study.

Although HFI reached the remarkable achievement of being cosmic background photon-noise-limited at the CMB peak frequency on intermediate and small angular scales (a few degrees to $\left.5^{\prime}\right)$, at very large angular scales $(\ell<15)$, the control of instrument systematic errors and foreground residuals took a long time to reach the required level to measure the reionization optical depth cosmological parameter with a signal-to-noise ratio (S/N) of 10 (Pagano et al. 2020). The noise and systematic effects residuals on the largest angular scales are nevertheless still far from the detector white-noise level needed to accurately remove the high-frequency foreground emission. Removing those from differential detector data requires use of the Solar System kinetic dipole. The solar dipole, common to all detectors and frequencies, is the strongest measurable differential signal at large scales. Improving its determination is therefore a key issue throughout this paper and the solar dipole is extracted from various splits of the HFI data (frequencies, Galactic masks, component separation models). This provides a tool for intercalibration of different instruments and missions but can also be used to 
constrain a better model of the high-frequency foreground systematic errors at large scales. These improvements, not achieved in the Planck Legacy release, are introduced in this work where we develop a new interstellar dust model (the dominant foregrounds for the CMB above $100 \mathrm{GHz}$ ) that is self-consistent with better $\mathrm{CMB}$ anisotropies and the associated better solar dipole measurement.

The paper is organized as follows: Sect. 2 describes the context and goals of the paper; Sect. 3 describes the algorithm to coherently extract the parameters and template maps of the foreground emission and the map zero levels, and presents the resulting dust model; Sect. 4 builds a self-consistent map of CMB anisotropies and solves for solar dipole parameters; and Sect. 5 summarizes our findings and conclusions.

\section{Context and definitions}

The relative motion between the Planck spacecraft and the CMB rest frame generates a dipolar modulation of the CMB intensity field. The spacecraft motion with respect to the cosmological background composed of two components. The first component, referred to as the orbital dipole, is caused by the relative velocity of the spacecraft at the L2 Lagrange point following the earth motion with respect to the Solar System rest frame. It is known with high accuracy at any time. This orbital dipole of order $30 \mathrm{~km} \mathrm{~s}^{-1}$ does not project on the sky maps. It is used to calibrate the absolute photometric response of each detector with a high-signal-to-detector-noise ratio of order $10^{5}$ at the 100 and $143 \mathrm{GHz}$ CMB-dominated channels, and $5 \times 10^{3}$ at $353 \mathrm{GHz}$ (see Planck Collaboration III 2020, hereafter HFI-Legacy).

The second component, referred to as the solar dipole, is the consequence of the relative velocity of the Solar System with respect to the cosmological rest frame. Although it is an order of magnitude larger than the orbital dipole, it cannot be determined from extragalactic astronomy, and therefore cannot help to calibrate the absolute response of the detectors. The dipole signal associated with this second component projects onto the sky maps with the same spectral energy distribution (SED) as the CMB cosmological anisotropies. As such, it is not a cosmological effect, but is a systematic effect in the maps extracted by component separation methods based on their different SEDs. The dipole signal is nevertheless not separable from the intrinsic dipolar term of the CMB primordial anisotropies. Using a $\Lambda \mathrm{CDM}$ model, measurement of the CMB anisotropies leads to an expected dipole amplitude of the order of $30 \mu \mathrm{K}$. This induces an uncertainty of the order of one percent on the kinetic Solar System velocity. This dipolar term is therefore included in the definition of the solar dipole, imposing a null dipolar term for the total CMB anisotropies maps.

The Solar System velocity measurement is independent of the CMB monopole temperature, which is known with an accuracy of $3 \times 10^{-4}$ (Fixsen 2009). This introduces additional uncertainty when expressing the amplitude of the solar dipole in $\delta T_{\mathrm{CMB}}$ anisotropies. Nevertheless, for convenience in making use of map differences at different frequencies, all intensity contributions to the dipole amplitudes are expressed in CMB temperature. As such, the conversion between velocity and $\mu \mathrm{K}_{\mathrm{CMB}}$ depends directly on the CMB temperature, which is obtained by fitting a Planck function in the COBE-FIRAS data, which has been refined using a combination of the solar dipole direction from WMAP and the FIRAS full-spectrum measurements (Fixsen 2009). In this regard, using the solar dipole to estimate the $\mathrm{CMB}$ monopole temperature makes the conversion from velocity to $\mu \mathrm{K}_{\mathrm{CMB}}$ slightly dependent on the dipole. However, the difference induced by a new dipole determination leads to a negligible correction.

The amplitude of the solar dipole signal is proportional to the response of each detector to a CMB intensity signal and is therefore very useful for testing the $\mathrm{CMB}$ relative photometric calibration of different $\mathrm{CMB}$ detectors. Its amplitude of approximately $3 \mathrm{mK} \mathrm{CMB}$ is very large with respect to the sensitivity of the $143 \mathrm{GHz}$ map (the noise on the dipolar amplitudes is less than $\left.10^{-2} \mu \mathrm{K}\right)$. This $3 \times 10^{5}$ signal-to-noise ratio has been used to measure the relative CMB photometric calibration on single detector maps. The consistency of the relative CMB photometric calibration on single detector maps within a frequency has a noise-limited accuracy of $10^{-5}$. The inter-calibration between HFI frequency maps is at the $4 \times 10^{-4}$ level from 100 to $353 \mathrm{GHz}$ (HFI-Legacy). This work uses these sensitivities, and exploits the fact that all detectors measure the same CMB solar dipole (hereafter referred to as the consistency argument) to control the residuals for foreground emission on the largest angular scales.

The CMB map is obtained from the frequency maps using two properties of CMB anisotropies to separate it from the foreground emission: Planck function SED and Gaussian statistics. The extraction of solar dipole amplitudes measured at various frequencies and on different sky fractions is the most powerful tool to detect the foreground residuals. It was used for example with the HFI data, and revealed residuals behaving with frequency like a Galactic dust component as can be seen in Tables 7 and 8 of the HFI-Legacy. These residuals are obviously due to the dust foreground removal, and showed that introducing largescale dipolar and quadrupolar terms to account for the largescale SED variation significantly reduces these drifts for the highest frequencies and sky fraction used to fit the solar dipole.

The solar dipole was recognized as an important tool when discussing the high-latitude dust foreground and zero level of frequency maps in Planck Collaboration XI (2014). Here, the solar dipole is extracted with an improved version of the SRoll algorithm, SRoll2 (Delouis et al. 2019), which was used to produce the Planck Legacy HFI maps. This upgraded algorithm, named SRoll2 . 2, introduces the following improvements:

- Use of 143, 217, 353, 545, and $857 \mathrm{GHz}$ nonpolarized Spider Web bolometers (SWB) data, in addition to Polarized Spider bolometers (PSB) data. This allows reduced systematic effects for intensity-only maps, while avoiding being limited by signal to noise ratios.

- Removal of polarized parts of the signals due to the small parasitic sensitivity to polarization of SWBs detectors.

- Improved correction of the Analog-to-Digital Converters nonlinearities, bringing the associated residuals below detector noise levels, even at the largest angular scales.

- Computation of single detector maps removing the polarization part in the signal.

- Use of the full resolution for the bandpass leakage correction where the signal is very strong, keeping the low resolution of the foreground templates on the rest of the maps to avoid introducing extra noise.

- Removal of the cosmic infrared background (CIB) monopole (Lagache et al. 1999) and use of CMB anisotropy maps with zero monopole bold.

- Setting the initial zero level of the intensity maps to the zero level of the $21-\mathrm{cm}$ emission of the H I Galactic gas foreground.

SRol12 2 builds two types of single-detector intensity maps ${ }^{1}$ : (i) one where a correction of all foreground signal bandpass

These maps are available at http://sroll20.ias.u-psud.fr 
mismatch between detectors is applied, and (ii) another where no correction of the foreground signal bandpass mismatch is applied. These type (ii) maps, smoothed at a common $1^{\circ}$, are used in this work.

\section{Foreground sky model and zero level offsets}

The solar dipole amplitudes of Planck Collaboration Int. XLVI (2016) Table 2, are drifting away from the amplitude measured at 100 and $143 \mathrm{GHz}$ both for higher frequencies ( 217 to $545 \mathrm{GHz}$ ), and when increasing the sky fraction $f_{\text {sky }}$ used to fit the dipole. The increase of the amplitude errors with frequency follows a typical Galactic dust SED. Such variations were expected from previous work on the COBE-DIRBE data (Lagache et al. 1998). HFI-Legacy shows that this problem is mostly solved by introducing large-scale variations of the dust SED in the model. This result is obtained using the stability of the solar dipole direction, taking the $100 \mathrm{GHz}$ one as reference. The dipole direction (especially the longitude) drifts by $0.1^{\circ}$ but the parameters for the $545 \mathrm{GHz}$ still exhibit large shift in amplitude and direction.

These results indicate that the residuals from the dust emission removal are a major concern when extracting the solar dipole above $100 \mathrm{GHz}$ in two ways:

- first, indirectly by the dust residuals along the Galactic ridge. This forces the introduction of a Galactic ridge mask $M_{\mathrm{g}}$ where the CMB anisotropies cannot be removed sufficiently accurately. This induces an unavoidable error when the dipolar term of the cosmological anisotropies is set to zero on the full sky (see Sect. 4.2);

- second, the large-scale Galactic emission in the part of the sky where the solar dipole is fitted outside the Galactic ridge mask affects the residuals of the component separation process. We therefore introduce terms in our foreground dust emission sky model that improve the modelling of the largescale SED variations.

The CMB anisotropies component of the maps, as well as the noise and systematic effects, are defined with a null monopole term. The HFI frequency maps do not have an absolute zero setting calibration. Any error in the zero level changes the contrast between the high- and low-galactic-latitude foreground emission, and therefore changes the foreground maps, especially where the intensity is very low at high latitude. The large-scale residuals of dust emission depend strongly on the accuracy of these zero levels, and we introduce consistency constraints from the solar dipole to refine the accuracy of the foreground model.

To start with, the initial setting of the zero level of the Planck Collaboration HFI frequency maps uses the method in which the extrapolated zero-intensity emission of the Galactic foreground gas corresponds to the extrapolated zero column density of gas (Planck Collaboration VIII 2014). The first step is performed by regressing the $857 \mathrm{GHz}$ emission, which is fully dominated by the dust emission intensity, to the neutral hydrogen interstellar gas column density. The other frequencies are then regressed to the $857 \mathrm{GHz}$ emission brightness. This sets the initial zero levels of the frequency maps with a sufficiently good accuracy as long as one does not require highly accurate intensity on very large scales. To improve on these results, and especially on the dust template map zero level, a more accurate method is developed here and these initial values need only be checked for compatibility with the ones extracted in this work ${ }^{2}$.

\footnotetext{
2 The full schematics of the algorithm is available at http:// sroll20.ias.u-psud.fr
}

\subsection{Dust model definition}

We decompose the intensity sky model as:

sky model $\sim \mathrm{CMB}_{\text {tot }}+$ Free-Free + Synchrotron $+\mathrm{CO}+$ dust.

In Eq. (1), there is only one extragalactic component, the CMB anisotropies. The CIB large-scale $(\ell<100)$ anisotropies are negligible with respect to the Galactic dust component, and are included in it. The first term $\mathrm{CMB}_{\text {tot }}$ is the total $\mathrm{CMB}$ anisotropies map containing the solar dipole and the primary cosmological anisotropies. The $\mathrm{CMB}_{\text {tot }}$ intensity is described by a single map of $\delta T_{\mathrm{CMB}}$ at all frequencies.

The three next terms of Eq. (1) are the low-frequency foregrounds: free-free, synchrotron emission, and the $\mathrm{CO}$ emission lines. In intensity, the free-free and synchrotron emissions are respectively one and two orders of magnitude smaller than the $\mathrm{CMB}$ anisotropies at $100 \mathrm{GHz}$. The free-free emission, which is only one order magnitude lower than the dust at $100 \mathrm{GHz}$, benefits from a stable and accurate SED. Furthermore, the synchrotron emission is lower than other emissions by orders of magnitude at higher frequencies. Therefore, free-free and synchrotron emissions are removed in an open loop using Planck Legacy results (Planck Collaboration X 2016). In the HFI data, the $\mathrm{CO}$ line emission is derived using only the large differences of response between detectors within a frequency band containing a CO line, and the Planck Legacy CO template maps used are detector noise limited. Nevertheless, their amplitudes are updated consistently with the new dust model.

The last term of Eq. (1), the interstellar dust emission, is the dominant foreground at frequencies above $100 \mathrm{GHz}$, and is described by a new model:

Dust $=f(v)$ Dust $+\frac{\partial f(v)}{\partial \beta} \Delta$ Dust $_{1}+\frac{\partial^{2} f(v)}{\partial \beta^{2}} \Delta$ Dust $_{2}$,

where the SED $f(v)=\operatorname{Planck}$ function $(v, T) \times\left(\frac{v}{v_{0}}\right)^{\beta}$, with $v_{0}$ being the reference frequency of the Dust map.

The temperature $T$ and the index $\beta$ are highly correlated when fitted in the range of frequencies used in this work, and therefore the temperature is taken as constant, and the SED variations are only described by the variations of the $\beta$ parameter. Given the available number of degrees of freedom (five frequencies), this has been shown to be acceptable in the HFI frequency range from previous work using a broader frequency range from the absolute intensity maps from COBE. The single temperature adopted, $18 \mathrm{~K}$, has been shown to give good SED fits for the H I dominant phase of the interstellar medium but also for the subdominant H II, which show very similar SED (Lagache et al. 1998).

The novelty of this dust model is the use of an analytical description of the averaged SED, and the introduction of two additive corrections modeling the effects of the SED spatial variations.

The first term of Eq. (2), Dust, is the initial dust map (see further), propagated from the frequency $v_{0}$ to each single detector map with a frequency $v_{\mathrm{b}}$ with the mean SED. Furthermore, the frequency is taken at the effective frequency that takes into account the difference between the response of the detector to a dust SED and the response to the CMB orbital dipole signal used to calibrate the detector with high accuracy in $\mathrm{K}_{\mathrm{CMB}}$.

The two other terms of Eq. (2) describe the spatially variable part of the SED, absorbing all differences to the spatially constant SED first term. The analytical description of the SED $f(v)$ allows a description of these variations as an expansion in 
terms of its first and second derivatives with respect to $\beta$. The model is not strictly described by the analytical expansion of the SED function $f(v)$ because the second spatial template $\Delta$ Dust $_{2}$ is independent of the first one, $\Delta$ Dust $_{1}$, which describes the firstorder expansion term. These two independent full sky maps, with a null average, have a resolution of order $1^{\circ}$ (HEALPix; Górski et al. 2005; $N_{\text {side }}=128$ ).

The initial dust map Dust is built from a map at sufficiently high frequency $\left(v_{0}=545 \mathrm{GHz}\right)$ that it only needs to be cleaned from the $\mathrm{CMB}$, the $\mathrm{CO}$, the free-free emission, and the synchrotron emission in an open loop. The CMB large-scale cosmological anisotropies maps taken from the Planck Legacy release, once propagated to $v_{0}$, are then three orders of magnitude smaller than the dust emission. The effects of the difference between the initial CMB used at this stage and the CMB map obtained ultimately in this work is therefore negligible.

From Eq. (2), we obtain the dust map model $\mathcal{D}\left(v_{\mathrm{b}}\right)$ for a single detector (bolometer $b$ ) map:

$\mathcal{D}\left(v_{\mathrm{b}}\right)=\frac{f\left(v_{\mathrm{b}}\right)}{f\left(v_{0}\right)}\left(\right.$ Dust $_{v_{0}}+\ln \left(\frac{v_{\mathrm{b}}}{v_{0}}\right) \Delta$ Dust $_{1}+\left(\ln \left(\frac{v_{\mathrm{b}}}{v_{0}}\right)\right)^{2} \Delta$ Dust $\left._{2}\right)$.

This equation is valid for maps expressed in spectral energy density. The 545 and $857 \mathrm{GHz}$ maps are calibrated on planets and are dealt with in spectral energy density expressed in $\mathrm{MJy} \mathrm{sr}^{-1}$. Nevertheless, $100-353 \mathrm{GHz}$ detectors calibrated on the orbital dipole of the CMB in $\delta T_{\mathrm{CMB}}$ are expressed in $\mu \mathrm{K}_{\mathrm{CMB}}$, and must be converted to spectral energy density. The of lower frequency CMB maps, offsets, and solar dipole amplitudes are finally converted back to $\mu \mathrm{K}_{\mathrm{CMB}}$ for the convenience of comparison with similar maps in the literature.

We know that SED variations exist at intermediate to large scales due to the gradients of (i) stellar radiation field intensity changing with Galactic radius and thus changing the dust temperature; and (ii) the relative fraction on each line of sight of the molecular, atomic, and ionized gas fraction, which are known to have slightly different dust properties. The two SED variation components are therefore defined by maps smoothed at $1^{\circ}$.

\subsection{Extraction of the CO emission lines, dust model parameters, and templates}

The SRoll2. 2 single detector intensity maps are built after removing the polarized signal and the bandpass mismatch coefficients are computed using template dust maps different from those build in the present work. We therefore use the set of maps in which the color correction has not been performed in order to be able to extract band pass mismatch coefficients coherent with the dust model using an iterative process.

We use $I_{\mathrm{b}}$ to denote the single detector intensity map built from the bolometer $b$ data. The effective frequency, $v_{\mathrm{b}}$, computed from the bandpass ground measurements is used in the first iteration of the processing and is corrected at each iteration when regressing the single detector map against the dust initial template Dust $v_{v_{0}}$.

The dust emission is the only broad-band foreground component not fully described by its SED, but is modeled empirically for the frequencies $v \geq 100 \mathrm{GHz}$ as a map with an average SED, completed with two additive correction maps describing the spatially variable SED. These three maps are extracted in this work using constraints other than previous component separations as discussed above (e.g., consistency of the solar dipole parameters). The $\mathrm{CO}$ and dust foregrounds being partially correlated, the $\mathrm{CO}$ foreground map is built using a $\mathrm{CO}$ template map from the Planck Legacy results (Planck Collaboration X 2016) but whose coefficients are extracted coherently with our new dust model.

Finally, the data contain noise and monopole offsets that are not directly measurable by the HFI differential detectors, and which are to be solved for using consistency arguments and data redundancies. Equation (4) defines the residual $R_{\mathrm{b}}$ which only contains the $\mathrm{CMB}_{\text {tot }}$ and the foreground components to be modeled; it is computed for each single detector map $I_{\mathrm{b}}$ from which the low-frequency foreground maps -taken from the Planck legacy maps as described above- are removed in an open loop.

$R_{\mathrm{b}}=I_{\mathrm{b}}-$ Free-Free $_{\mathrm{b}}-$ Synchrotron $_{\mathrm{b}}-O_{\mathrm{HI}}$.

The initial offset $O_{\mathrm{HI}}$ applied to the single detector map sets the zero level of the single-detector intensity maps $I_{\mathrm{b}}$, following Planck Collaboration VIII (2014), which sets this zero level to the extrapolation to zero column density of $\mathrm{HI} 21-\mathrm{cm}$ emission of interstellar gas at high latitudes. This is a first approximation of the absolute zero level of the dust foregrounds. The addition of the ionized component traced by $\mathrm{H} \alpha$ emission changes the zero level by $3 \%$ within the uncertainties, and the molecular component is negligible at high Galactic latitudes. The offsets are further refined in this work with smaller uncertainties, and the final results should be consistent with the initial offsets and their uncertainties. The offset values for detectors, either within the same frequency band or with different frequencies, change the contrast of the large scales at high latitude but are constrained by the solar dipole signal which is a common component to all bolometers.

For the 100 to $353 \mathrm{GHz}$ detectors, the single detector map offset $O_{\mathrm{b}}$ is adjusted in three steps:

1. The first step uses all pair differences, which gives offsets with respect to a zero average offset of all these bolometers.

2. The second step adjusts the average offset using the fact that the solar dipole vector seen by all the detectors is the same.

3. The third step is the determination of the $545 \mathrm{GHz}$ frequency map offset, performed by minimizing its projected effect by the dust emission SED from $545 \mathrm{GHz}$ to the lower frequency offsets. The initial dust template is extracted from the $545 \mathrm{GHz}$ frequency map, which is corrected at the end of this step. This provides a new dust template Dust ${ }_{v_{0}}$, which is used at the next iteration.

The residual $R_{\mathrm{b}}$ also contains the nonrecoverable noise and systematic effects residuals $N_{\mathrm{b}}$ (including the very small negligible residuals following the low-frequency foreground emission removal). In order to improve the solar dipole measurement, an improved model of the two main partially correlated highfrequency foreground emission components is built, simultaneously extracting the $\mathrm{CO}$ line emission intensity coefficients $\gamma_{\mathrm{b}}$, the effective frequencies $v_{\mathrm{b}}$, and template maps $\Delta$ Dust $_{1}$ and $\Delta$ Dust $_{2}$ describing the new thermal emission dust model. For each sky pixel seen by all pairs of bolometers $b 1$ and $b 2$, the constraint used is to minimize the difference $\Delta R_{\mathrm{b} 1, \mathrm{~b} 2}=R_{\mathrm{b} 1}-R_{\mathrm{b} 2}$, expressed in $\mu \mathrm{K}_{\mathrm{CMB}}$. The $\mathrm{CMB}_{\text {tot }}$ term cancels and

$$
\begin{aligned}
\Delta R_{\mathrm{b} 1, \mathrm{~b} 2}= & \mathcal{D}\left(v_{\mathrm{b} 1}\right)-\mathcal{D}\left(v_{\mathrm{b} 2}\right)\left(\gamma_{\mathrm{b} 1}-\gamma_{\mathrm{b} 2}\right) \widetilde{\mathrm{CO}} \\
& +O_{\mathrm{b} 1}-O_{\mathrm{b} 2}+N_{\mathrm{b} 1}-N_{\mathrm{b} 2},
\end{aligned}
$$

where $\mathcal{D}\left(v_{\mathrm{b}}\right)$ is the dust emission (Eq. (3)). The initial dust map $\mathcal{D}\left(v_{0}\right)$ is computed from the SRoll2 . $2545 \mathrm{GHz}$ map after subtracting in an open loop CO, free-free, and CMB. For bolometer $b$, the specific projection coefficient $f\left(v_{\mathrm{b}}\right) / f\left(v_{0}\right)$ from the 
template dust map computed from the $545 \mathrm{GHz}$ bandpass map accounts for the detector response to the dust SED through the effective frequency $v_{\mathrm{b}}$. These effective frequencies, initially set to the values computed from the ground measurements of the bandpass (Planck Collaboration IX 2014), are adjusted simultaneously with the $\gamma_{\mathrm{b}}$ coefficients.

The CO Planck Legacy maps are used as a template to extract a single effective set of $\gamma_{\mathrm{b}}$ coefficients defining the CO lines response at $100,217,353 \mathrm{GHz}$. The fact that there is no CO line in the $143 \mathrm{GHz}$ frequency band is used to regularize the solution, requiring that the detectors have a negligible response correlated with the $\widetilde{\mathrm{CO}}$ template. Weaker interstellar molecular lines nevertheless exist in the $143 \mathrm{GHz}$ frequency band, but this affects the $\mathrm{CO}$ coefficients by an error of $1.1 \%$, inducing an error on the solar dipole amplitude of $0.06 \mu \mathrm{K}$ and $10^{-4}$ degrees in direction, which is negligible.

The offsets $O_{\mathrm{b}}$ are strongly degenerate with the large-scale SED variation of the dust, and are therefore also solved for simultaneously using the same minimization of the differences $\Delta R_{\mathrm{b} 1, \mathrm{~b} 2}$ used to compute the large-scale SED variation of the dust. Based on the internal stability of the frequency maps, the quantity to minimize, $b$, for each pixel is $p$ :

$$
\begin{aligned}
\chi_{\mathrm{hf}}^{2}=\sum_{(b 1, b 2)} \sum_{p}\left(\Delta R_{\mathrm{b} 1, \mathrm{~b} 2, \mathrm{p}}-\mathcal{D}\left(v_{\mathrm{b} 1}\right)+\mathcal{D}\left(v_{\mathrm{b} 2}\right)\right. \\
\left.-\left(\gamma_{\mathrm{b} 1}-\gamma_{\mathrm{b} 2}\right) \widetilde{\mathrm{CO}}_{\mathrm{p}}-O_{\mathrm{b} 1}-O_{\mathrm{b} 2}\right)^{2} .
\end{aligned}
$$

Conditions on the mean parameter values between all single detectors are needed to minimize Eq. (6), which is based on map differences. This is possible thanks to several closure conditions.

- For $O_{\mathrm{b}}, \Delta \mathrm{Dust}_{1}$, and $\Delta \mathrm{Dust}_{2}$, we impose that the mean values be null.

- For $\gamma_{\mathrm{b}}$, we force the mean values to be null at $143 \mathrm{GHz}$.

- The level of residual dust emission in the mean map over all detectors after removing the dust and the $\mathrm{CO}$ model, and an initial CMB anisotropies map, is related to incorrect estimation of the mean $f\left(v_{\mathrm{b}}\right)$. Setting this level to zero provides a closure condition for all $v_{\mathrm{b}}$. At the first iteration, we use the CMB SMICA map.

From this minimization, we obtain:

- the correction to the response to a dust SED for bolometers calibrated on the CMB orbital dipole as an effective frequency $v_{\mathrm{b}}$

- the additive SED spatial variation described by the $\Delta$ Dust $_{1}$ and $\triangle$ Dust $_{2}$ maps,

- the CO effective coefficients $\gamma_{\mathrm{b}}$, and

- the relative offsets $O_{\mathrm{b}}$ of the single detector maps.

Furthermore, a global $100-353 \mathrm{GHz}$ offset $\bar{O}$ must be introduced to adjust the offsets of the $100-353 \mathrm{GHz}$ maps with respect to the unknown $545 \mathrm{GHz}$ map offset. Moreover, considering the large uncertainty on the $545 \mathrm{GHz}$ map offset, a correction must also be introduced. This will correct the large-scale initial dust map template taken from the $545 \mathrm{GHz}$ frequency map. This is performed by adding a constraint on the solar dipoles built for each detector (Sect. 3.3).

\subsection{Determination of the frequency map offsets}

The SED spatial variations of the dust are strongly degenerate with the average zero levels of the frequency maps. Equation (6) minimization only constrains the relative adjustments of these offsets, and does not affect the initial band average zero levels of the frequency maps which have rather large uncertainties with respect to the relative ones, especially for the 353 and $545 \mathrm{GHz}$ frequency bands.

A global (100 to $353 \mathrm{GHz}$ ) offset $\bar{O}$ is introduced in the algorithm. This is determined thanks to the fact that the spatial distortions of the dust foreground initial dust map uncertainties bias the solar dipole vector directions. Thus, minimization of the dispersion of the single detector CMB solar dipole vector over the $43(100$ to $353 \mathrm{GHz}) \mathrm{HFI}$ bolometers allows us to compute $\bar{O}$.

To remove the solar dipole, the cosmological CMB anisotropies map must be removed from the $\mathrm{CMB}_{\text {tot }}$ map. Nevertheless, this CMB anisotropies map, which is extracted by component separation methods, is not reliable in the narrow Galactic ridge that needs to be masked and filled with a constrained realization within a Galactic ridge mask $M_{\mathrm{g}}$ before setting the dipole term to zero on the whole sky. The mask $M_{\mathrm{g}}$ is defined as the sky contained between two symmetrical latitudes centered on the Galactic plane and characterized by the sky fraction that it covers, $f_{\text {sky }}$.

The CMB anisotropies full-sky map, denoted $\mathrm{CMB}_{M_{\mathrm{g}}}$, is the $\mathrm{CMB}_{\text {tot }}$ map from a component separation, in which the dipole term has been set to zero bold after masking with a Galactic ridge mask $M_{\mathrm{g}}$. This induces biases that depend on the size of the Galactic mask $M_{\mathrm{g}}$, and on the procedure used to fill this mask with $\mathrm{CMB}$ anisotropy-constrained realizations. Before we have a $\mathrm{CMB}_{\text {tot }}$ anisotropies map consistent with the new foreground model, we use, at the first iteration, the Planck Legacy CMB SMICA map, which is estimated to be reliable for cosmology outside a 5\% Galactic mask. At the second and following iterations, a CMB large-scale anisotropies map coherent with the results of Sect. 3 can be built. From all single intensity bolometer maps we remove $R_{\mathrm{b}}$ from 100 to $353 \mathrm{GHz}$, the current dust model and $\mathrm{CO}$ template, and the adjustments of the offsets obtained in Sect. 3.2. The weighted average over all bolometers provides the new current $\mathrm{CMB}_{\text {tot }}$.

A new three-component (CMB, dust, and $\mathrm{CO}$ ) separation algorithm named Bcsep is was developed using the difference between single detector maps in nested iterative loops, which also uses the consistency of the solar dipole parameters between frequencies. This requires us to start with adequate approximations and the convergence of the algorithm is the test of this condition. This new component separation is performed by subtracting the dust or the CMB maps from the frequency residual map $R_{\mathrm{b}}$ and iteratively improving $\mathrm{CMB}_{\mathrm{tot}}$ and the three maps of the dust model per frequency:

$$
\mathrm{CMB}_{\text {tot }}=\left(\sum_{\mathrm{b}} \frac{1}{\sigma_{\mathrm{b}}^{2}}\right)^{-1} \sum_{\mathrm{b}} \frac{1}{\sigma_{\mathrm{b}}^{2}}\left(R_{\mathrm{b}}-\mathcal{D}\left(v_{\mathrm{b}}\right)-\gamma_{\mathrm{b}} \widetilde{\mathrm{CO}}-O_{\mathrm{b}}-\bar{O}\right),
$$

where $\sigma_{\mathrm{b}}^{2}$ is the variance of the cleaned maps for $f_{\text {sky }}=0.9$ estimated by simulations.

Equation (8) defines a residual map $\boldsymbol{\aleph}_{\mathrm{b}, M_{\mathrm{g}}}$ as the single detector intensity map $R_{\mathrm{b}}$ after removal of the high-frequency foreground emission model, the current CMB anisotropies $\mathrm{CMB}_{M_{\mathrm{g}}}$, and the relative offsets extracted previously. $\boldsymbol{\kappa}_{\mathrm{b}, M_{\mathrm{g}}}$ is therefore the map containing only the CMB solar dipole, together with the noise and residuals from the foregrounds removal:

$$
\begin{aligned}
\aleph_{\mathrm{b}, M_{\mathrm{g}}} & =R_{\mathrm{b}}-\mathrm{CMB}_{M_{\mathrm{g}}}-\mathcal{D}_{\mathrm{b}}-\gamma_{\mathrm{b}} \widetilde{\mathrm{CO}}-O_{\mathrm{b}} \\
& =A_{\mathrm{b}, M_{\mathrm{g}}, \bar{O}} \sum_{\ell=1, m=0, \pm 1} \mathcal{A}_{\mathrm{b}, M_{\mathrm{g}}} \mathcal{Y}+\bar{O}+N_{\mathrm{b}, M_{\mathrm{g}}},
\end{aligned}
$$

where 
- $A_{\mathrm{b}, M_{\mathrm{g}}, \bar{O}}$ is the solar dipole amplitude extracted from $\mathrm{CMB}_{M_{\mathrm{g}}}$,

- $\mathcal{A}_{\mathrm{b}, M_{\mathrm{g}}, \bar{O}}$ are the three $a_{\ell, m}$ vector components $\left(a_{1,0}, a_{1,1}\right.$ real and imaginary parts) of the solar dipole direction,

- $\mathcal{Y}$ are the three spherical harmonics $Y_{\ell=1, m}$ component maps of the solar dipole, and

- $\bar{O}$ is the global offset of the detector maps $100-353 \mathrm{GHz}$ initially set to zero.

To constrain $\bar{O}$, we impose that all detectors show a minimal dispersion of the solar dipole direction vector. Starting with $\bar{O}=$ 0, we loop on Eqs. (8)-(10) and solve for the $\bar{O}$ value. The solar dipole parameters for bolometer $b$ are fitted within a set of ten masks $\mathcal{M}$ with smaller and smaller areas identified by their $f_{\text {sky }}$ and taken in the sky region left outside the Galactic ridge $M_{\mathrm{g}}$.

For any given detector, the minimization Eq. (9) extracts a running $\bar{O}$ and mask $M_{\mathrm{g}}$, the dipole amplitude $A_{\mathrm{b}, M_{\mathrm{g}}, \bar{O}}$, and direction vector $\mathcal{A}_{\mathrm{b}, \mathcal{M}_{\mathrm{p}}, \bar{O}}$ by minimizing the difference taken over for a mask $\mathcal{M}$ between the residual map $\boldsymbol{\aleph}_{\mathrm{b}, \mathrm{p}, M_{\mathrm{g}}}$ map and the model solar dipole map plus the current $\bar{O}$ :

$\chi_{\mathrm{b}, \mathcal{M}, M_{\mathrm{g}}}^{2}=\sum_{\mathrm{p}} \mathcal{M}_{\mathrm{p}}\left(\boldsymbol{\aleph}_{\mathrm{b}, \mathrm{p}, M_{\mathrm{g}}}-A_{\mathrm{b}, M_{\mathrm{g}}, \bar{O}} \sum_{\substack{\ell=1 \\ m=0, \pm 1}} \mathcal{A}_{\mathrm{b}, \mathcal{M}_{\mathrm{p}}, \bar{O}} \mathcal{Y}_{\mathrm{p}}-\bar{O}\right)^{2}$

Here, $\bar{O}$ is then carried back into Eq. (8) if we change $M_{\mathrm{g}}$ or into Eq. (9) if we change only $\mathcal{M}$ after the optimization of $M_{\mathrm{g}}$, until convergence.

This should not be affected at the first iteration by the choice of the CMB cosmological anisotropies SMICA map in the first iteration as it converges to a stable $\bar{O}$ value:

$\chi_{\mathrm{b}, M_{\mathrm{g}}}^{2}=\sum_{b=1}^{N_{\mathrm{b}}} \sum_{\mathcal{M}=1}^{N_{\mathcal{M}}}\left(\mathcal{A}_{\mathrm{b}, \mathcal{M}_{\mathrm{p}}, \bar{O}}-\sum_{b=1}^{N_{\mathrm{b}}} \sum_{\mathcal{M}=1}^{N_{\mathcal{M}}} \frac{\mathcal{A}_{\mathrm{b}, \mathcal{M}_{\mathrm{p}}, \bar{O}}}{N_{\mathcal{M}} N_{\mathrm{b}}}\right)^{2}$.

At this stage, the $545 \mathrm{GHz}$ map initial offset uncertainty still indirectly affects the zero levels at all lower frequencies through the distortion of the initial dust template and the minimization of Eqs. (6)-(10), and directly by the propagation of the $545 \mathrm{GHz}$ offset residual to lower frequencies through the SED $f(v)$.

We define the 100 to $353 \mathrm{GHz}$ offsets $O_{v}$ as the average of $\left(O_{\mathrm{b}}+\bar{O}\right)$ over the frequency band $v$. The correction of the initial offset at $545 \mathrm{GHz}, O_{545}$, which also affects all the $O_{v}$ (following the $f(v) / f\left(v_{0}\right)$ emission law), is the one which minimizes Eq. (11):

$\chi^{2}=\sum_{v=100}^{353} \frac{1}{\sigma_{\mathrm{HI}}^{2}}\left(O_{v}-\left(\frac{f(v)}{f\left(v_{0}\right)}\right) O_{545}\right)^{2}$,

where $\sigma_{\mathrm{HI}}$ is the rms of the initial zero level determination uncertainties at frequency $v$. This correction therefore mainly affects the $353 \mathrm{GHz}$.

The following section (Sect. 3.4) discusses the coherence of the offset determination.

\subsection{Absolute zero level correction consistency}

The absolute zero levels of the maps are critical in order to identify the foreground emission that needs to be modeled with the correct zero level. In Sects. 3.2 and 3.3, we develop a method to constrain these offsets using the solar dipole strong signal that should be seen in all single detector maps with the same direction vector.

Four map offsets are determined in sequence:
Table 1. Zero level offset for each correction step.

\begin{tabular}{|c|c|c|c|c|c|}
\hline \multirow[b]{2}{*}{$\begin{array}{c}\text { Frequency } \\
{[\mathrm{GHz}]}\end{array}$} & \multirow{2}{*}{$\begin{array}{c}\text { Planck } \\
O_{\mathrm{HI}} \\
{[\mu \mathrm{K}]}\end{array}$} & \multicolumn{4}{|c|}{ This work } \\
\hline & & $\begin{array}{c}O_{\mathrm{b}} \\
{[\mu \mathrm{K}]}\end{array}$ & $\begin{array}{c}+\bar{O} \\
{[\mu \mathrm{K}]}\end{array}$ & $\begin{array}{c}\text { Final } \\
{[\mu \mathrm{K}]}\end{array}$ & $\begin{array}{l}\text { rms } \\
{[\mu \mathrm{K}]}\end{array}$ \\
\hline 100 & $-3.5 \pm 3.6$ & -3.6 & 1.14 & 1.05 & 1.0 \\
\hline 143 & $-8.28 \pm 2.8$ & -1.08 & 3.67 & 3.49 & 1.1 \\
\hline 217 & $-26.1 \pm 5.8$ & -2.87 & 1.87 & 1.24 & 2.4 \\
\hline \multirow[t]{2}{*}{353} & $-142.4 \pm 27$ & 6.26 & 11.0 & 6.17 & 12.2 \\
\hline & {$\left[\mathrm{MJy} \mathrm{sr}^{-1}\right]$} & & & {$\left[\mathrm{MJy} \mathrm{sr}^{-1}\right]$} & {$\left[\mathrm{MJy} \mathrm{sr}^{-1}\right.$} \\
\hline 545 & $-0.884 \pm 0.012$ & & & 0.0048 & 0.0023 \\
\hline 857 & $-0.534 \pm 0.16$ & & & -0.124 & 0.04 \\
\hline
\end{tabular}

Notes. For the HFI frequency bands, $O_{\mathrm{HI}}$ gives the initial map offsets based on the zero $\mathrm{HI}$ column density extrapolation; $O_{\mathrm{b}}$ gives the relative bolometer offsets averaged over the frequency band; column $+\bar{O}$ gives the offsets after adding the global offset $\bar{O}$; column Final gives the final offsets after removing the $545 \mathrm{GHz}$ offset. The last column gives the final uncertainty on these zero levels.

1. The initial setting of the zero levels in the SRoll2.2 maps $O_{\mathrm{HI}}$ are computed following Planck Collaboration VIII (2014). Their uncertainties, although small in the CMB frequencies, affect the large-scale dust emission at high latitudes at 353 and $545 \mathrm{GHz}$.

2. Equation (6) describes the computation of the relative offsets of the 100 to $353 \mathrm{GHz}$ single bolometer maps, $O_{\mathrm{b}}$. The constraint is the consistency between all pairs of single bolometer maps. To allow the minimization based on differences between maps, the mean of all these relative offsets per single bolometer is set to zero.

3. Section 3.3 deals with the zero level mean correction over all 100 to $353 \mathrm{GHz}$ bolometers, $\bar{O}$. This correction is obtained by minimizing the variance of the solar dipole directions over all single detectors and masks using Eq. (9). The single bolometer solar dipoles are extracted using Eq. (10). Finally, we measure $\bar{O}=4.74 \mu \mathrm{K}$. The relative corrections are bigger on the lowest frequencies.

4. Finally, Eq. (11) adjusts the $545 \mathrm{GHz}$ offset, $O_{545}$, by minimizing the lower frequency offset corrections affected by the projection of $O_{545}$ following the dust SED $f(v)$. This offset is applied to the initial dust map Dust $v_{545}$, and we iterate over the whole process.

The results after convergence are reported in Table 1. The data zero level offset corrections, averaged per HFI frequency band, are given as cumulative offset values obtained after each correction of the algorithm refining the initial offset $O_{\mathrm{HI}}$. The convergence of the iterative process shows the stability of the algorithm which leads to a minimal dispersion of the solar dipole vector direction for the single-detector $\mathrm{CMB}$ anisotropy maps in the frequency bands $100-353 \mathrm{GHz}$.

The final absolute zero level uncertainties are computed using a set of ten simulations for the four lower frequencies. Those simulations have representative noise and systematic effects but no attempt is made to quantify the uncertainties due to the dust model using simulations because we have no reliable statistical model. These uncertainties at 100,143 , and $217 \mathrm{GHz}$ are almost equal to the dispersion of the single bolometers at each frequency. Thus, these errors are probably dominant over the instrumental systematic effect dispersion. The uncertainty at $353 \mathrm{GHz}$ is about one-third of the dispersion between bolometers which indicates that the zero level correction reflects the dust foreground effects. The uncertainty at $545 \mathrm{GHz}$ is computed 

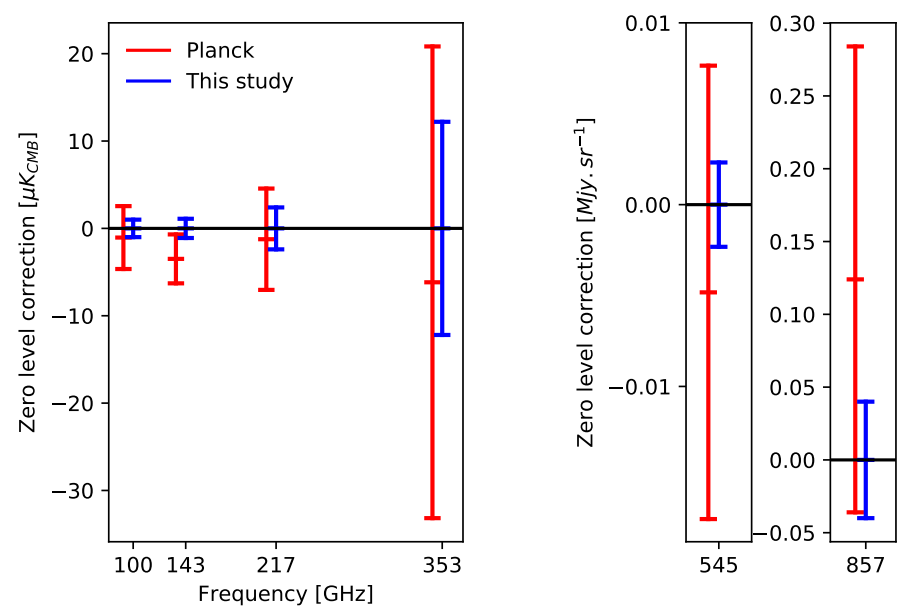

Fig. 1. Zero levels of the maps corrections: the initial one in red, and the final cumulated offset corrections taken as reference in blue, with their uncertainties.

using a Monte Carlo approach based on uncertainties at the lower frequencies. The $O_{545}$ correction induces a marginally significant correction at $353 \mathrm{GHz}$ only. These correction steps are weakly correlated, and the convergence confirms that the algorithm steps improve the offsets efficiently.

Figure 1 displays the map offset results as a function of the frequency bands and the initial ones from Planck in red; the final corrections from this work taken for reference, in blue, are consistent with the initial frequency map offsets but with an accuracy that is better by factors of 2.5 to 5 .

The $857 \mathrm{GHz}$ is obtained in Appendix A extending the dust model beyond $545 \mathrm{GHz}$ and using the solar dipole constraint. This is independent of the lower frequency results.

\subsection{Dust model results}

Figure 2 presents these additive correction maps (as Healpix maps at $N_{\text {side }}=128$ ) to take into account the spatial variation of the dust emission SED. These spatial variation maps in the right column are built from $\Delta$ Dust $_{1}$ and $\Delta$ Dust $_{2}$ maps, and are weighted by the frequency-dependent ratios $\partial f(v) / \partial \beta$ and $\partial^{2} f(v) / \partial \beta^{2}$ respectively. These maps are compared with the HFI-Legacy ones (left column), where each frequency SED spatial variation map has been determined independently but only for dipole and quadrupole terms. The large scales are similar although obtained in different ways, and despite the fact that the initial dust maps were taken from different frequencies: $857 \mathrm{GHz}$ for HFI-Legacy and $545 \mathrm{GHz}$ for this work.

The SED spatial variations are described by an expansion in $\ln \left(v_{\mathrm{b}} / v_{0}\right)$ at the second order, but are associated with two independent large-scale maps providing more degrees of freedom. The two bottom maps in Fig. 3 show these additive correction maps for the $143 \mathrm{GHz}$ frequency band. The top map shows an estimate of an effective $\beta$ corresponding to the total correction of the two terms.

For a more quantitative comparison, Fig. 4 shows the ratio of the power spectra of the first- and second-order additive SED correction maps at $143 \mathrm{GHz}$, with different $f_{\text {sky }}$.

There is a strong Galactic center anti-center dipole term $(\ell=1$ and 3$)$, and weaker latitude pattern $(\ell=2$ and 4$)$, and also a white power distribution, probably reflecting the random interstellar cloud distribution. The low rise at $\ell>15$ shows the noise contribution. For $f_{\text {sky }}=0.82$, the first-order SED variation

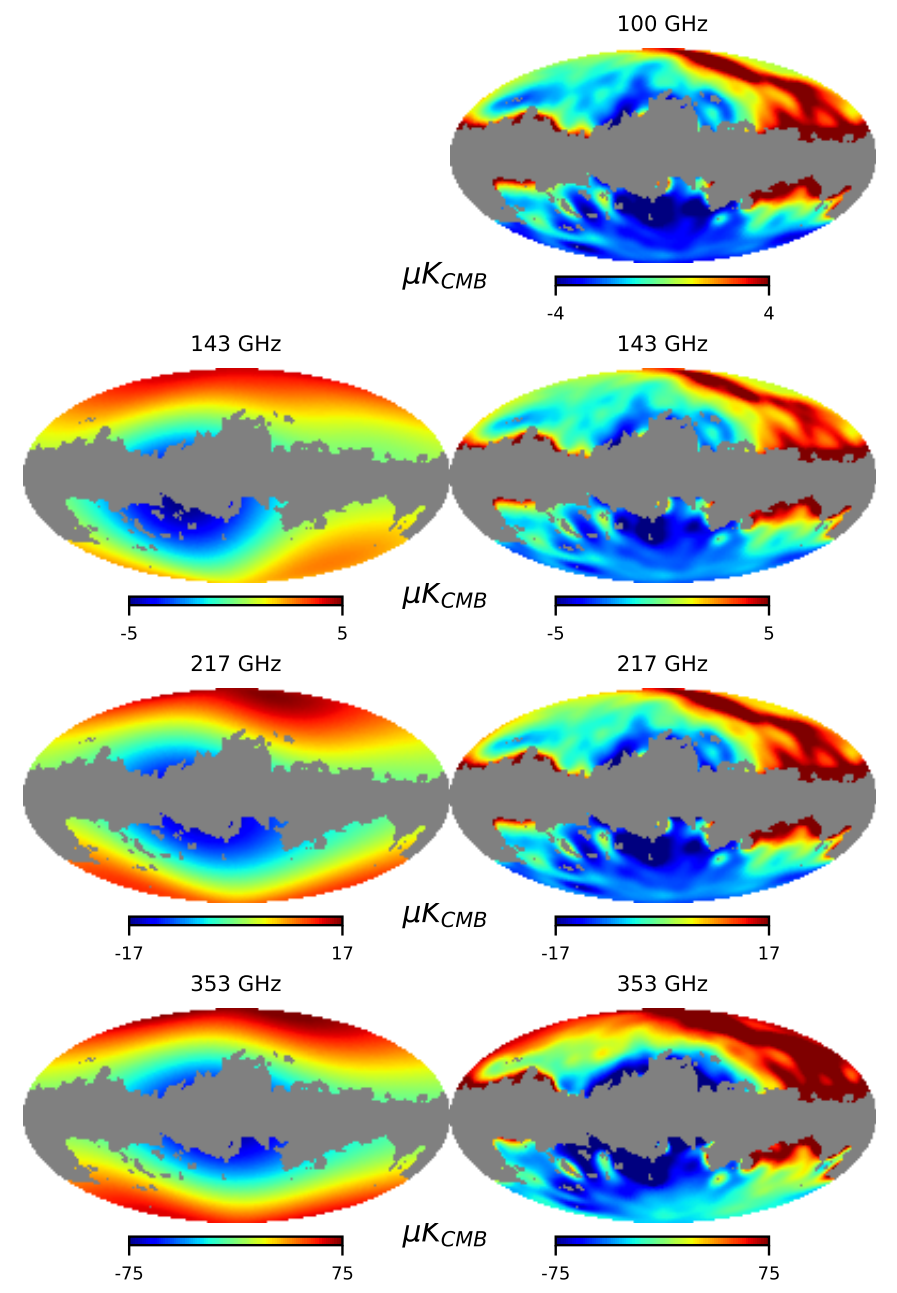

Fig. 2. Additive correction maps from this work (right). The equivalent correction maps from HFI-Legacy, which only contain dipole and quadrupole terms, are shown in the left column. The $100 \mathrm{GHz}$ map is not displayed as it was used as the reference for the dipole direction.

corrections show a strong center-to-anticenter dipole term at the $30 \%$ to $50 \%$ level of the dust initial spectra with opposite sign to the dust map. The second-order correction is two orders of magnitude smaller (one order of magnitude in the map). This agrees with the astrophysical assumption that the dust SED gradients are induced by large-scale Galactic physics -gradient of gas molecular fraction in latitude and star light energy density gradient with Galactic radius-, and not small-scale variations of the dust properties which are seen in the flat power spectrum $(5<\ell<15)$.

To test the validity of the dust model above $545 \mathrm{GHz}$, we describe its extension to $857 \mathrm{GHz}$ in Appendix A.

\section{Cosmic microwave background large-scale results and solar dipole determination}

The solar dipole is extracted from each single detector $\mathrm{CMB}_{\text {tot }}$ map from which we can subtract $\mathrm{CMB}$ anisotropies. The first iteration uses the Planck Legacy CMB anisotropies SMICA map after masking the Galactic ridge, keeping $95 \%$ of the sky as recommended in Planck Collaboration IV (2020). In later iterations, the Bcsep $\mathrm{CMB}_{\text {tot }}$ map from Eq. (8) is used. 


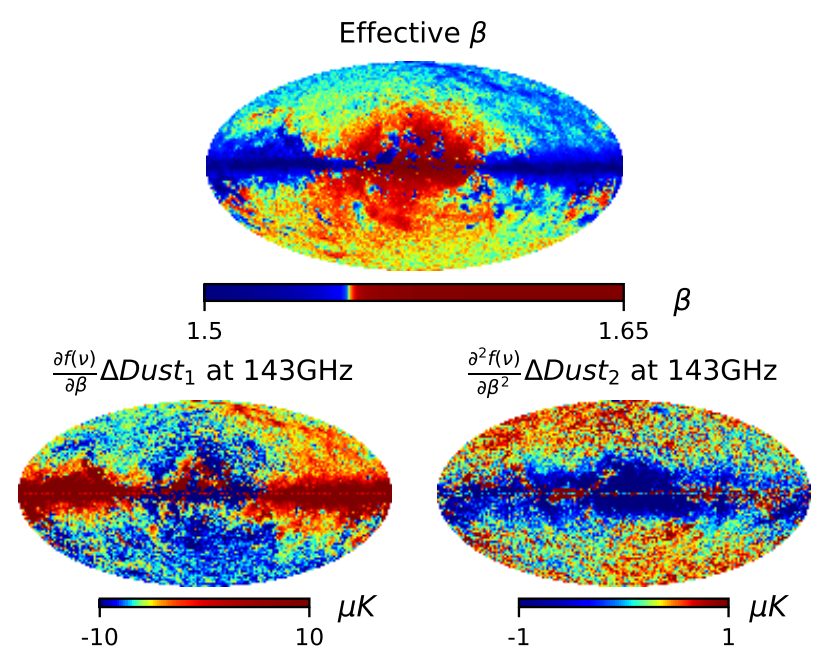

Fig. 3. Effective dust parameter $\beta$ variation map (top). In the narrow Galactic ridge, a single $\beta$ correction per line of sight is not meaningful considering the large variations of the SED. Bottom maps: two independent intensity correction maps associated with the first and second $\beta$ order derivative.

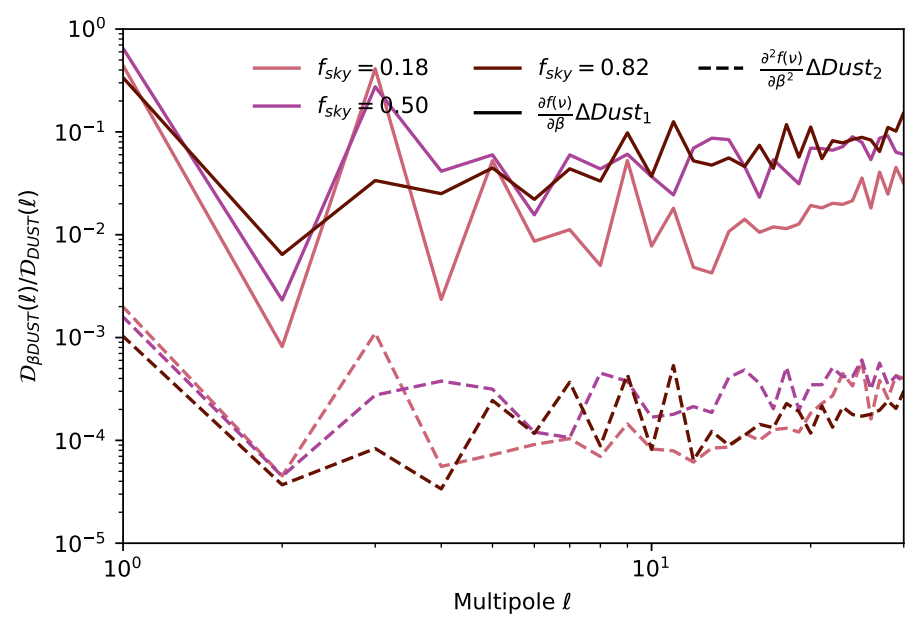

Fig. 4. Power spectrum ratio of the $\Delta$ Dust $_{1}$ term to the dust emission (continuous lines) for different high latitude masks. The dashed lines show the power spectrum ratio of the $\Delta$ Dust $_{2}$ term to the dust emission. These results are given for the emission at $143 \mathrm{GHz}$.

\subsection{New cosmic microwave background large-scale anisotropies map}

To illustrate the accuracy of the CMB cosmological anisotropies map built by the Bcsep method, we use an end-to-end simulation which contains CMB realizations, noise, systematic effect residuals, and also a single $\mathrm{CO}$ model and a dust model as per Eq. (2) which is removed at the end of the processing. Thus, this simulation does not intend to evaluate the Bcsep ability to solve for the dust model, but only for the noise and systematic effect residuals.

Figure 5 shows the difference between the simulated input map of CMB primary anisotropies (without solar dipole) and the retrieved one. The narrow Galactic ridge towards the inner galaxy is the only visible contributor to a significant dipole term, with a main vector direction toward $b=l=0^{\circ}$. To avoid this bias, the solar dipole is extracted outside the Galactic ridge mask $M_{\mathrm{g}}$. The error induced on the solar dipole amplitude is $0.103 \mu \mathrm{K}$ with $f_{\text {sky }}=0.90$, and $0.108 \mu \mathrm{K}$ with $f_{\text {sky }}=0.95$. This shows that

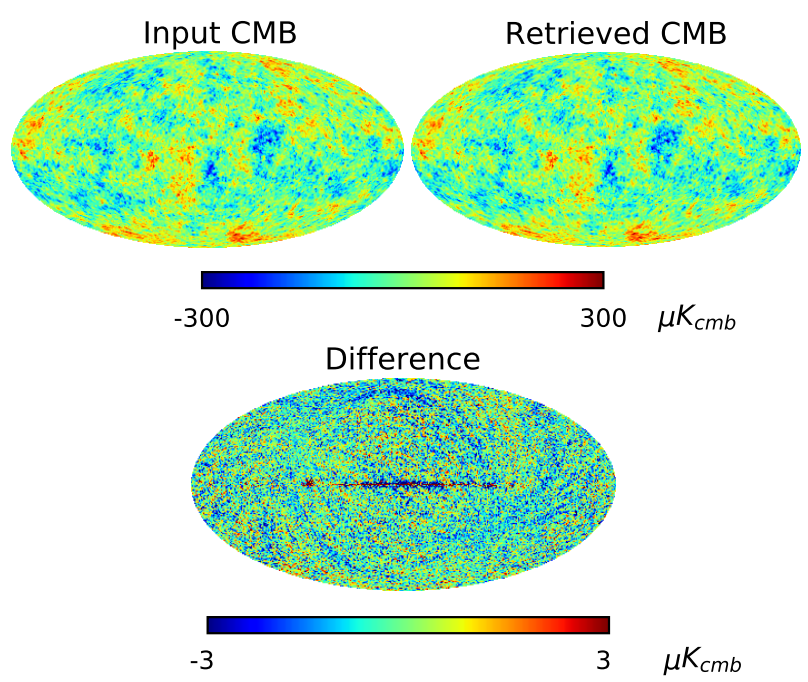

Fig. 5. Retrieved primordial CMB anisotropies Bcsep map compared to the input CMB (top maps). Bottom map: difference between input and output with a color scale that is 100 times smaller.

the removal of noise and systematic effects on the CMB maps outside $M_{\mathrm{g}}$-within this range of $f_{\mathrm{sky}}$ - is not a direct problem for the solar dipole determination. The dust foreground residuals remain the main issue.

Figure 6 displays the difference maps between the $\mathrm{CMB}_{\text {tot }}$ maps obtained by Bcsep and the four Planck Collaboration methods Commander, NILC, SEVEM, and SMICA (Planck Collaboration IV 2020). The residuals are much bigger than in Fig. 5 and are dominated by the Galactic foreground emission residuals at low latitudes. At high Galactic latitude $\left(b>20^{\circ}\right)$, NILC and SMICA, which do not depend on a physical model of the Galactic components to extract the Gaussian $\mathrm{CMB}$, are in very good agreement. The difference map between SMICA and Bcsep at $b>20^{\circ}$ does not show significant residuals either. All differences containing either the Commander or the SEVEM maps show Galactic residuals at $b>20^{\circ}$ and zodiacal dust emission residuals along the ecliptic equator.

We note that the $\mathrm{CMB}_{\text {tot }}$ Bcsep map is only aimed at a significant improvement at $\ell<30$ and outside the bright Galactic ridge $M_{\mathrm{g}}$ where it has smaller large-scale dust residuals than the Planck legacy maps. Bcsep does not intend to build a CMB map for analysis of cosmological parameters mostly based on CMB power spectra at $\ell>30$, and is not characterized for that purpose.

\subsection{Optimization of the $C M B$ anisotropies removal}

Lacking a model to build statistically representative and controlled dust foreground maps, the foreground residuals cannot be fully evaluated by simulations. A semi-quantitative approach is nevertheless possible by comparing data and simulations. The behavior of the solar dipole parameters with frequency and sky fraction are sensitive probes to test both the method for removing $\mathrm{CMB}$ anisotropies and that for removing Galactic foreground residuals.

Large-scale foreground residuals in the part of the sky where the dipole is fitted induce a source of systematic effects on the dipole parameter retrieval. These effects are introduced in the simulation by adding an empirical estimate of the foreground residuals defined as the difference map from two-component separation method: SMICA-Bcsep shown in Fig. 6. 


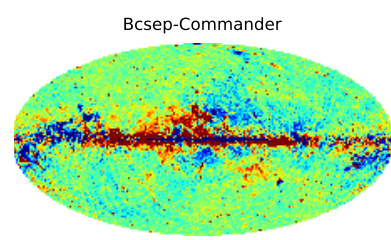

Commander-NILC

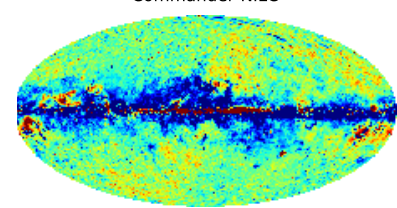

NILC-SEVEM

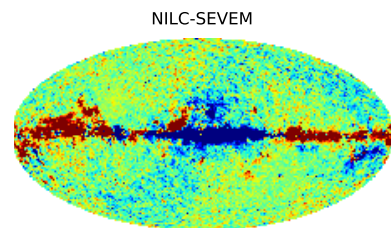

SEVEM-SMICA

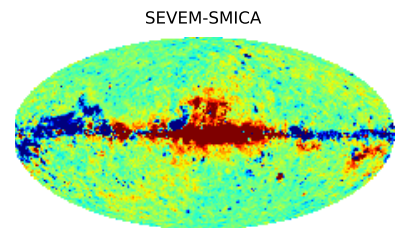

Bcsep-NILC

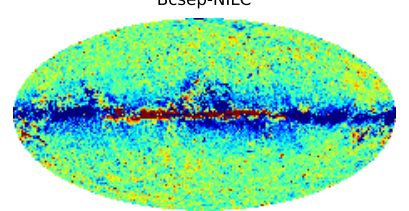

Commander-SEVEM

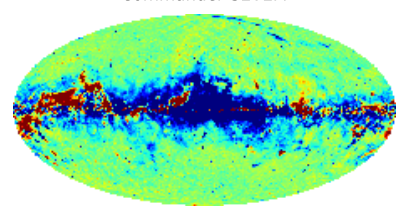

NILC-SMICA

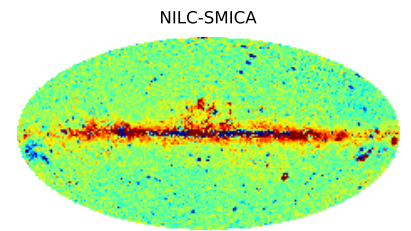

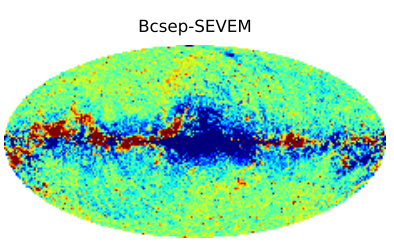

Commander-SMICA

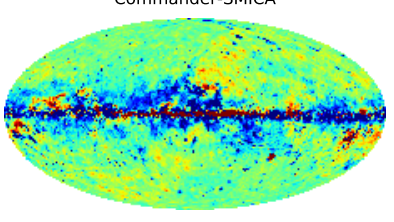

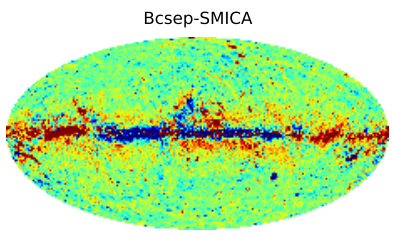

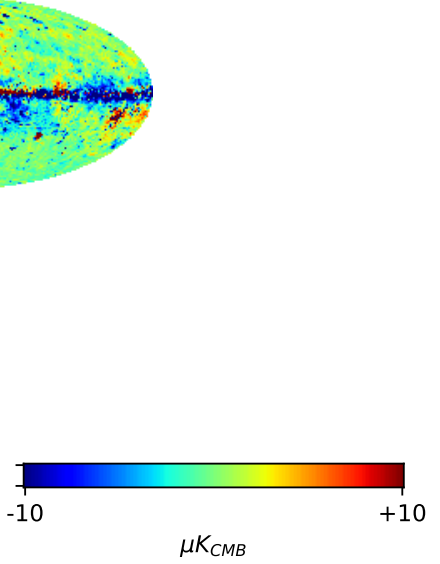

Fig. 6. Difference between the CMB maps built by Bcsep and the four Planck Collaboration component separation methods.

We now use the $\mathrm{CMB}_{\text {tot }}$ Bcsep map built in Sect. 4.1. When extracting the solar dipole from the full sky $\mathrm{CMB}_{\text {tot }}$, the solar dipole parameters are biased by the Galactic ridge residuals seen in Fig. 6. This leads to the use of the Galactic ridge mask $M_{\mathrm{g}}$ where the $\mathrm{CMB}_{\text {tot }}$ is affected by large Galactic residuals. This mask $M_{\mathrm{g}}$ is filled with a simulated CMB constrained at the boundaries (Wandelt et al. 2004; Thommesen et al. 2020). By definition, the all-sky map of the CMB cosmological anisotropies has no dipolar term, and is built by removing the dipolar term from the $\mathrm{CMB}_{\text {tot }}$ map. The solar dipole is thus fitted in a mask $\mathcal{M}$ taken as the complement of $M_{\mathrm{g}}$. On the one hand, the masked sky replaced by a constrained CMB induces an uncertainty on the dipole parameters. This uncertainty is evaluated by the dispersion of the retrieved solar dipole parameters from 1000 Monte Carlo realizations changing the $f_{\text {sky }}$ fraction of the Galactic ridge mask $M_{\mathrm{g}}$. On the other hand, the bias induced by the dust residuals in the central Galactic ridge decreases with increasing $M_{\mathrm{g}}$ sky fraction. The combination of these two effects presents a minimum, which defines the optimal. The large-scale dust residuals in the $\mathrm{CMB}_{\text {tot }}$ map also slightly affect the dipole measurement (Sect. 4.1). Any detectable dust residual effect will then appear as a frequency and/or an $f_{\text {sky }}$ dependence of the dipole parameters when changing $M_{\mathrm{g}}$.

We want to compare these two effects: the large-scale SED spatial variations of the dust emission, and the $\mathrm{CMB}_{\text {tot }}$ affected by the filling of $M_{\mathrm{g}}$. These behaviors, which can be observed in the data, are simulated. Figure 7 shows the computed solar dipole parameters for the $100-353 \mathrm{GHz}$ frequencies, when varying the Galactic ridge mask $M_{\mathrm{g}}$ in which the $\mathrm{CMB}_{\text {tot }}$ Bcsep map is filled with constrained $\mathrm{CMB}$ realizations. The dipole parameters are plotted as a function of the sky fraction left outside $M_{\mathrm{g}}$ used to fit the dipole.

At first, the systematic effects affecting the retrieved dipole parameters are tested both on simulations and data to ensure that the simulations are a good representation of the data. The two first rows in Fig. 7 use the processing algorithm without SED variation correction. The input solar dipole parameters for the simulated data are taken from the final determination from this work, and given by the black lines with gray bands. In these two first rows, we see the remaining effects of the dust SED variations (included in the simulations and not corrected for), which are well simulated when compared to the results obtained using the data. The magnitude and sign of the residuals qualitatively demonstrate the quality of the simulation, even though the dust behavior is not quantitatively accurate, as expected, lacking a reliable physical model of the dust SED variations. In the third and fourth rows of Fig. 7, the algorithm now includes the dust SED spatial variation correction. Both show a spectacular reduction of the previous trends. This demonstrates that the simulation takes into account the SED variations with the right order of magnitude, and also that the algorithm captures them well.

The dipole term has to be removed from the $\mathrm{CMB}_{\text {tot }}$ map, and is only well known outside a Galactic ridge mask $M_{\mathrm{g}}$ where there are significant Galactic dust residuals. This masking induces an error due to leakages between the very large-scale anisotropies and the dipole term. Figure 8 shows the results for the recovered parameters of the solar dipole as a function of the sky fraction left outside of the varying $M_{\mathrm{g}}$ where the dipole is fitted. The dispersion of the solar dipole parameters is evaluated by simulation with 1000 realizations of the CMB filling of $M_{\mathrm{g}}$. The plain gray wedges show the dipole term dispersion when nothing is done to compensate this leakage. The dashed (dotted) gray wedges show the dispersion of the dipole term after filling $M_{\mathrm{g}}$ with a smoothed $1^{\circ}\left(6^{\circ}\right) \mathrm{CMB}$ realization of the large scales only (up to $\ell=5$ ) constrained by the cosmological parameters HFI best-fit (Planck Collaboration V 2020), and continuity constraints at the boundaries. Once the dipole term is removed from the $\mathrm{CMB}_{\text {tot }}$ map, as per the adopted definition, it is used to remove the CMB 

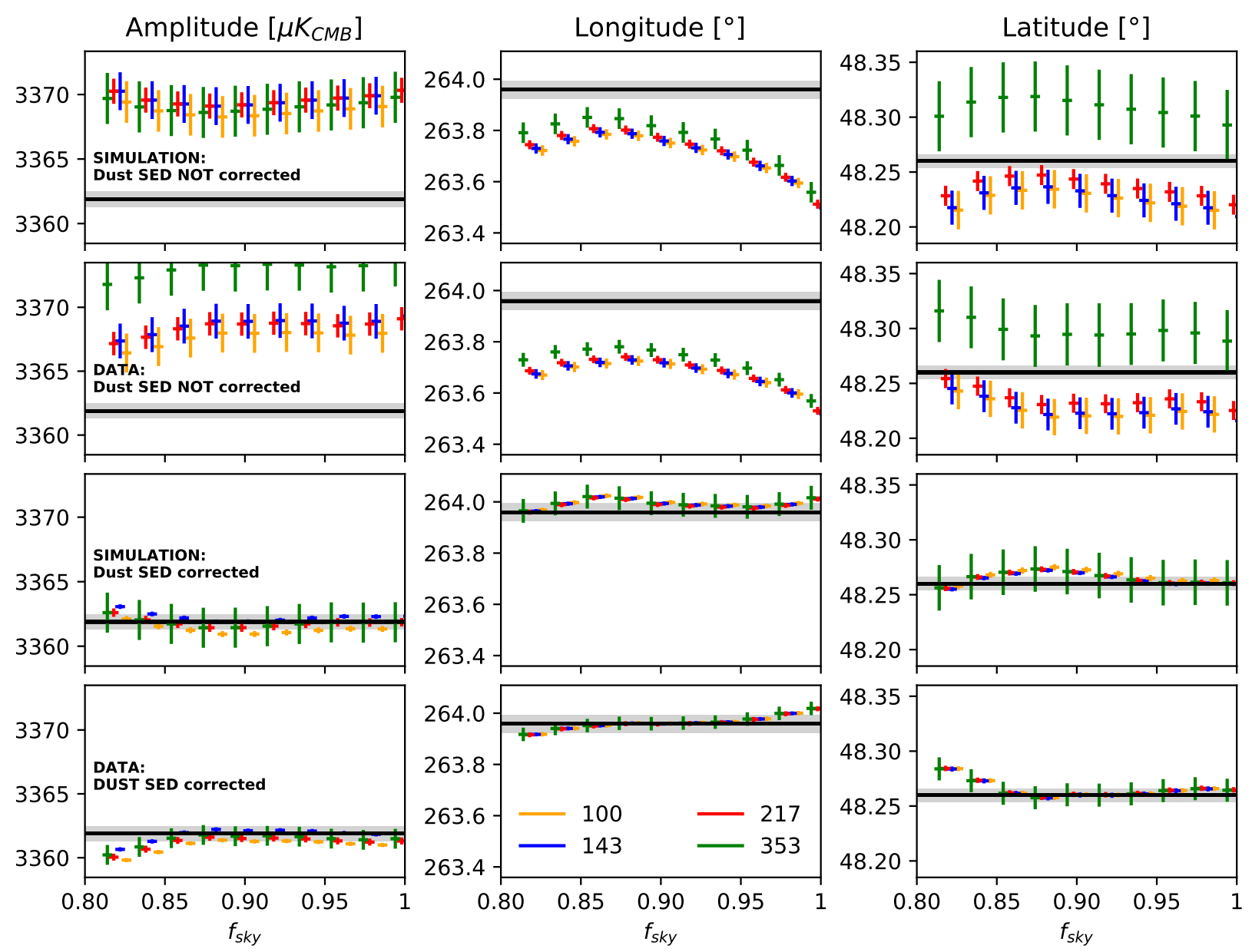

Fig. 7. Reconstructed solar dipole parameters along sky fraction used to remove the Galactic ridge from the retrieved CMB. Frequencies are color coded from 100 to $353 \mathrm{GHz}$. Black lines reflect the input dipole parameters (taken from the final result of this work). The gray band gives the uncertainties on these parameters.

anisotropies from the full sky map. The solar dipole is then fitted in $\mathcal{M}$. This removal and filling procedure introduces a nonrecoverable uncertainty, increasing with $M_{\mathrm{g}}$ estimated using results shown in Fig. 8.

The simulations with the qualitatively representative dust component residuals defined above show the expected bias in the amplitudes and in direction (longitude bias as expected larger than the latitude one) of the solar dipole parameters. The biases are maximal when the Galactic ridge mask $M_{\mathrm{g}}$ is null, and decrease with decreasing $f_{\text {sky }}$ following the predicted behavior. For no masking of the Galactic ridge, the direction is shifted by about $2^{\prime}$ in longitude. When $f_{\text {sky }}$ decreases to 0.95 , the longitude bias is reduced by a factor of two, and crosses the longitude dispersion for $1^{\circ}$ smoothing. For this sky fraction, the amplitude parameter shows a bias of $0.25 \mu \mathrm{K}$. The dispersion for $6^{\circ}$ smoothing crosses the bias for $f_{\text {sky }}=0.92$. We therefore conclude that, for the CMB anisotropies removal map, a $M_{\mathrm{g}}$ mask leaving $f_{\text {sky }}=0.95$ available for the fit of the dipole is optimal for the $\mathrm{CMB}$ anisotropies removal with a safer $1^{\circ}$ smoothing.

\subsection{Dipole fit stability}

Figure 9 is a zoom-in of the Fig. 7 third row displaying simulations for the four frequencies $100-353 \mathrm{GHz}$. The dipole parameters for all frequencies show the behavior described in Sect. 4.2 with a bias increasing for increasing $f_{\text {sky }}$ from $f_{\text {sky }}=0.8$ to 1.0 (purple line), and a dispersion, as the gray wedge ( $1^{\circ}$ smoothing), decreasing rapidly with increasing $f_{\text {sky }}$. The four frequencies show very similar patterns. The longitude, the most sensitive parameter, shows a minimal error for the optimal $M_{\mathrm{g}}$ size to $f_{\text {sky }}=0.95$ as expected. The behavior of the three parameters shows a correlated but nonmonotonic behavior as expected when dominated by the dispersion induced by the large-scale foregrounds for $f_{\text {sky }}<0.95$, within the range predicted by the gray wedge. It is at present impossible to draw high-quality statistically representative Galactic foreground emission maps and thus fully evaluate the foreground residuals. The uncertainties are given by the dispersion between detectors in each frequency band, and increase with frequency. Calibration errors resulting from noise and systematic effects, both evaluated by simulations, are visible as a variation in amplitude between frequencies with the right order of magnitudes.

\subsection{Solar dipole results}

We now wish to test the effect of the dust residuals on the stability of the solar dipole parameters, when increasing frequencies and varying $\mathcal{M}$, keeping the optimal $M_{\mathrm{g}}$ constant $\left(f_{\text {sky }}=0.95\right)$. Figure 10 shows the stability of the solar dipole parameters when the fitting mask $\mathcal{M}$ spanning from $f_{\text {sky }}=0.19$ to 0.9 (see Sect. 4.2). The stability of the solar dipole parameters is impressive, and they do not show any obvious trend with the dipole 


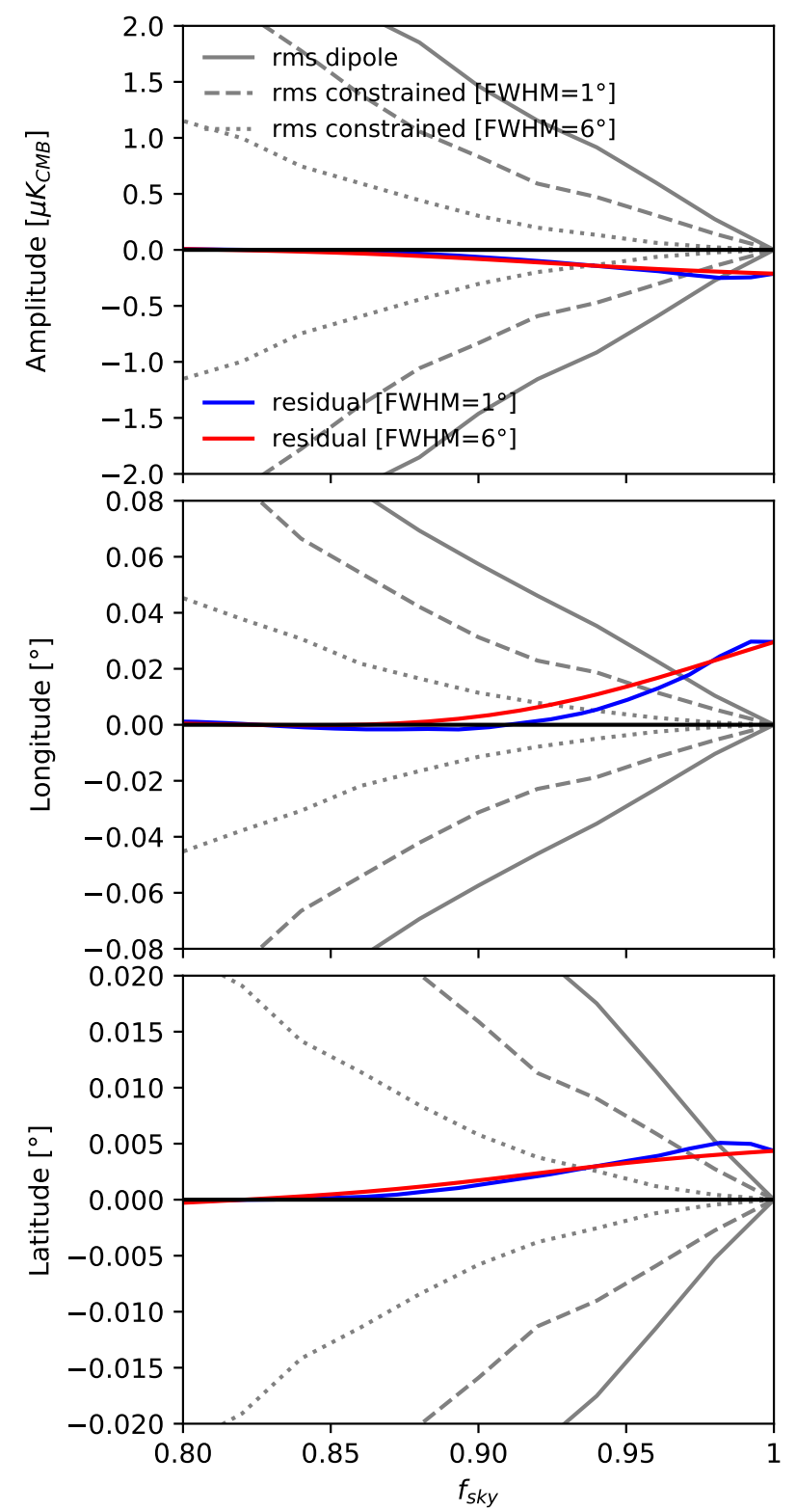

Fig. 8. Retrieved solar dipole parameters as a function of the sky fraction outside the Galactic ridge mask $M_{\mathrm{g}}$ from which the solar dipole is extracted. $M_{\mathrm{g}}$ is filled with a constrained realization of CMB anisotropies. The blue and red lines show the bias for two smoothing $\left(1^{\circ}\right.$ and $\left.6^{\circ}\right)$. The gray wedges show the dispersion of the solar dipole parameters.

fitting mask $\mathcal{M}$ nor with frequency. The most noticeable discrepancies are in amplitude and nearly all within $1 \sigma$. The final result of this paper (average over the three lower frequencies) is shown as black lines and gray band uncertainties. We therefore conclude that there is no dust foreground residuals detectable above the noise.

Table 2 shows the final results of the solar dipole parameters, computed as the weighted averaged per frequency over $\mathcal{M}$ with $f_{\text {sky }}=0.3$ to 0.8 . For amplitudes, the dispersion between detectors within a frequency band is a more relevant measure of the whole calibration process, including systematic effects. HFI-Legacy uses 100 full end-to-end simulations of the overall absolute calibration process based on the recovery of the solar dipole input. These simulations retrieve the rms uncertainties of the calibration measured by the solar dipole amplitude

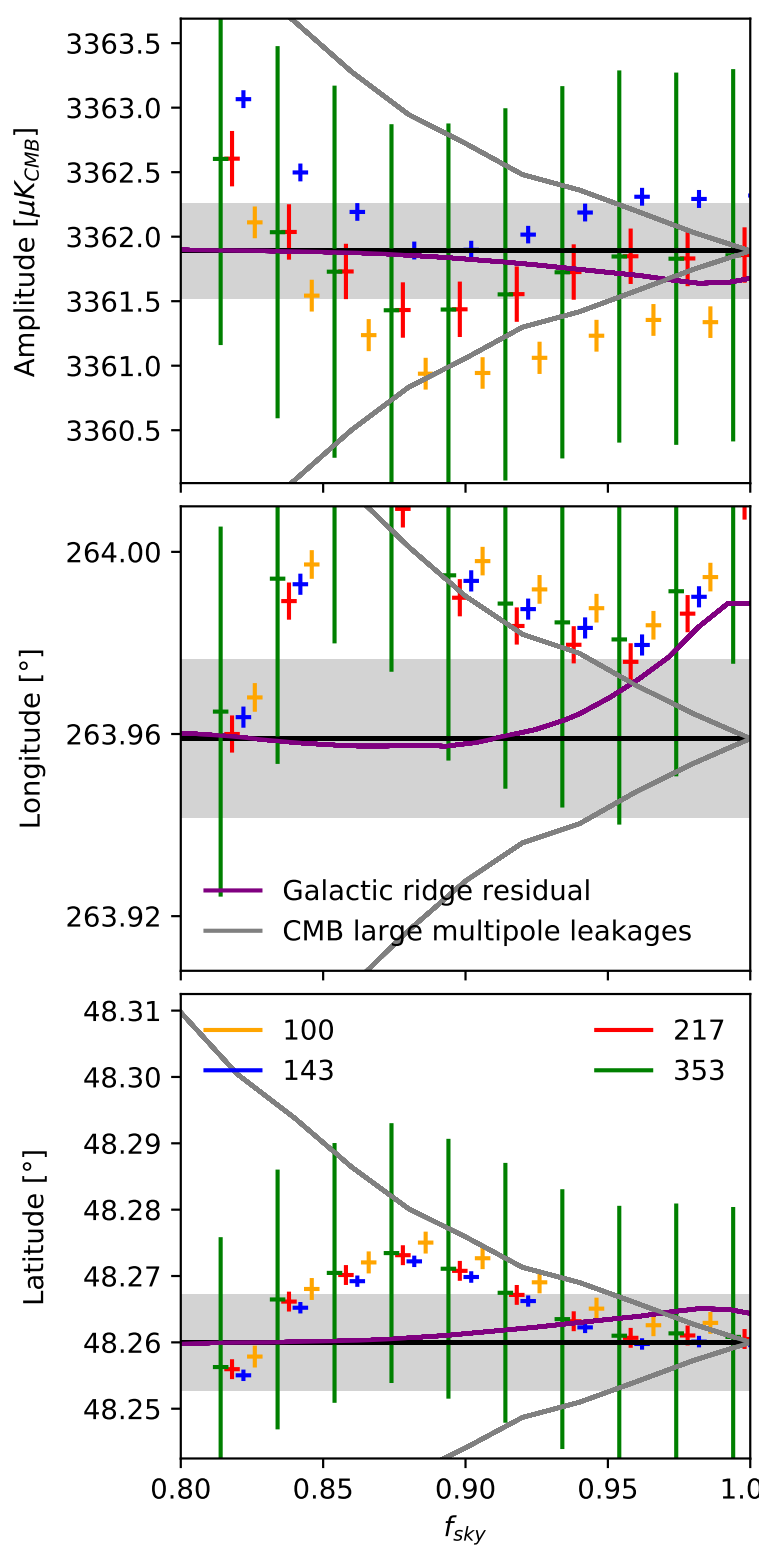

Fig. 9. Zoom-in of Fig. 7 for simulated data. The purple lines show the Galactic ridge emission residual after the Bcsep component separation. The gray lines show the dispersion of the effect of $M_{\mathrm{g}}$ filling.

rms: $1.5 \times 10^{-4}$ at frequency from 100 to $217 \mathrm{GHz}$ increasing to $4 \times 10^{-4}$ at $353 \mathrm{GHz}$. Our results in amplitude are coherent with the HFI-Legacy calibration mismatch estimates. Thus, the weighted average (AVG) of the 100, 143, and $217 \mathrm{GHz}$ results provides the best estimate of dipole parameters. The AVG amplitude uncertainty $(0.36 \mu \mathrm{K})$, computed as the dispersion between the three frequencies, is significantly larger than the intrafrequency uncertainties, but reflects the calibration error due the residual systematic effects.

For direction, the given uncertainties are the statistical errors. The HFI-Legacy results are obtained by alignment of the $143-353 \mathrm{GHz}$ frequencies solar dipole directions on the $100 \mathrm{GHz}$ solar dipole direction, assuming it was the best reference, because of its best combination of high CMB sensitivity and low foregrounds. In the present work, this assumption is replaced by the constraint of minimum dispersion of the dipole directions for all 100 to $353 \mathrm{GHz}$ detectors, a more refined dust model of the spatial variation of the SED, and coherent 


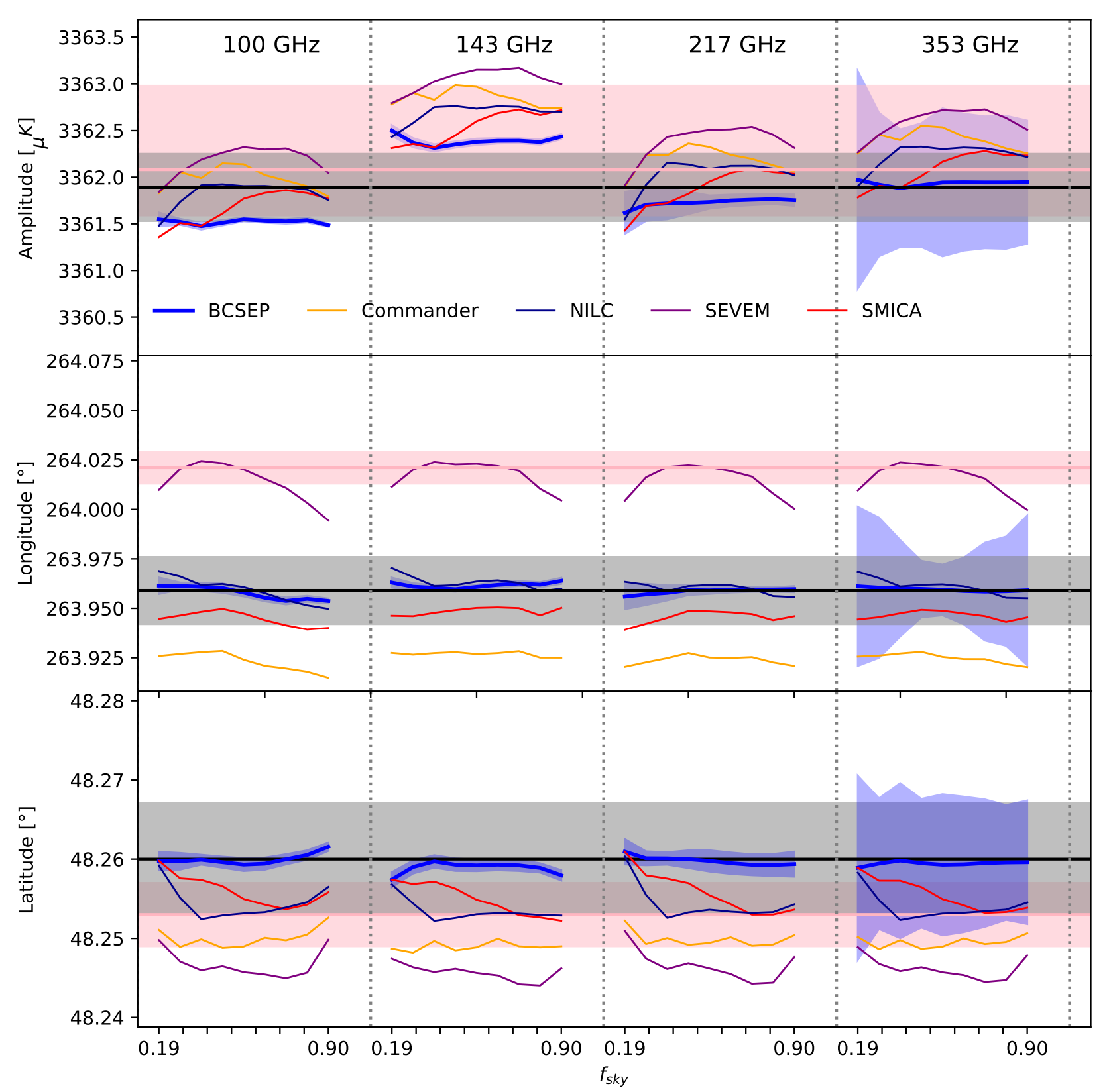

Fig. 10. Variation of the solar dipole parameters (in rows) for different frequency bands (in columns) as a function of the Galactic mask in which the dipole is fitted. The black lines and gray band uncertainties show the final results of this work, using the Bcsep CMB anisotropies map. For reference, the pink lines and bands show the HFI-Legacy measurements. The four Planck Legacy CMB anisotropies maps are also tested, and are shown color coded.

large-scale CMB anisotropies. We also introduce a better masking-filling procedure for the removal of the Galactic ridge residuals in the CMB cosmological anisotropies. Figure 10 compares the present results with the HFI-Legacy results, shown as the pink lines and bands. The results for the amplitude and latitude are highly compatible within their error bars. Nevertheless, the longitude is not, albeit very stable when varying $f_{\text {sky }}$.

An empirical test of the systematic effects related to the component separation is to use different CMB maps. This is done also in Fig. 10, and the four component separation methods used for the CMB anisotropies are shown with thin lines. Although using CMB from Commander, NILC, SEVEM, and SMICA is not fully consistent with the new and better foreground dust model developed in this work, the comparison remains interesting. When other component separations are used to get the CMB anisotropies, the stability with varying $f_{\text {sky }}(\mathcal{M})$ parameter is not as good as the one achieved by the Bcsep one. It strengthens the statement that the CMB removal, when done with an optimal Galactic ridge mask, has little effect on the solar dipole parameters fitted in a mask $\mathcal{M}$ covering a very broad range of sky fraction, thus showing no sign of large-scale dust residuals.

Thommesen et al. (2020) and Planck Collaboration Int. LVII (2020) increasing the Galactic mask up to $80 \%$ of the sky is far from optimal. The dispersion of the amplitude in these papers grows from $\pm 2.5 \mu \mathrm{K}$ for $M_{\mathrm{g}}$ in the range $f_{\text {sky }} 0.95-0.75$ and reaches $\pm 10 \mu \mathrm{K}$ for $f_{\text {sky }}=0.30$. These large uncertainties are induced when setting the dipole term to zero in a larger and larger Galactic mask. Conversely, the method described in the present work optimizes this mask and reduces the uncertainties by large factors, making full use of the CMB anisotropies that 
Table 2. Solar dipole parameters averaged per frequency and per fitting mask $\mathcal{M}$.

\begin{tabular}{lccc}
\hline \hline $\begin{array}{c}\text { Frequency } \\
{[\mathrm{GHz}]}\end{array}$ & $\begin{array}{c}A \\
{[\mu \mathrm{K}]}\end{array}$ & $\begin{array}{c}l \\
{[\mathrm{deg}]}\end{array}$ & $\begin{array}{c}b \\
{[\mathrm{deg}]}\end{array}$ \\
\hline 100 & $3361.52 \pm 0.04$ & $263.958 \pm 0.003$ & $48.260 \pm 0.001$ \\
143 & $3362.36 \pm 0.04$ & $263.961 \pm 0.002$ & $48.259 \pm 0.001$ \\
217 & $3361.74 \pm 0.11$ & $263.959 \pm 0.002$ & $48.260 \pm 0.001$ \\
353 & $3361.93 \pm 0.71$ & $263.959 \pm 0.019$ & $48.259 \pm 0.009$ \\
AVG & $3361.90 \pm 0.36$ & $263.959 \pm 0.003$ & $48.260 \pm 0.001$
\end{tabular}

Notes. AVG is the average over CMB-dominated frequencies 100, 143, and $217 \mathrm{GHz}$. The $353 \mathrm{GHz}$ is also given; it has larger uncertainties because of the much larger dust emission.

are accurately measured over up to $95 \%$ of the sky. We also note that the $4 \sigma$ calibration discrepancy between 100 and $143 \mathrm{GHz}$ mentioned in Table 10 of Planck Collaboration Int. LVII (2020) is of the same order as the absolute calibration reflected by the shift of the amplitude of the solar dipole by $4 \mu \mathrm{K}$ in their paper showing a photometric calibration problem.

Figure 11 shows three combinations of the solar dipole parameters, the results of this work, and the results of HFI-Legacy (in red). The longitude is the only discrepant parameter, being shifted by $-3.6^{\prime}$ of longitude $\left(2.5^{\prime}\right.$ on the sky) well outside the error ellipses. There is a key difference between the extraction method used in this work and the one used in HFI-Legacy. These latter authors fit the dipole parameters outside of a Galactic ridge mask for which the zero dipolar term of the CMB anisotropies has been removed without filling the mask with constrained CMB realizations uncontaminated by dust residuals. This induces a bias on the dipolar component in the part of the sky outside the Galactic ridge mask $M_{\mathrm{g}}$. This bias has been simulated on the present final maps by removing the step of the gap filling, and fitting the dipole parameters outside the optimal mask $M_{\mathrm{g}}$. The dipole direction is displayed as the blue cross and dotted error ellipse in the top panel of Fig. 11. This point falls near the HFI-Legacy red point, as expected as neither of them have optimal gap filling. The bias on the sky is $1.8^{\prime}$ when the shift observed is $2.5^{\prime}$. This simulation shows the bias induced when $M_{\mathrm{g}}$ is not filled with a constrained CMB, and we added this bias to the uncertainties of the legacy shown by the full blue line ellipse.

Averaging the three lowest Planck-HFI CMB frequencies (Table 2) with uncertainties including the systematic effects therefore leads to the final solar dipole parameters:

$A=[3361.90 \pm 0.04$ (stat.) \pm 0.36 (syst.) $] \mu \mathrm{K}$;

$l=263.959 \pm 0.003$ (stat.) \pm 0.017 (syst.);

$b=48.260 \pm 0.001$ (stat.) \pm 0.007 (syst.).

This result is consistent with HFI-Legacy for amplitude and latitude. The longitude of the dipole axis is shifted by $-2.5^{\prime}$ on the sky. This is fully explained by the difference in procedure, having introduced the filling of the Galactic ridge mask with constrained $\mathrm{CMB}$ anisotropy realizations before setting its dipolar term to zero.

\section{Conclusion}

Table 3 summarizes the progress made in terms of the accuracy of the determination of the solar dipole parameters. This
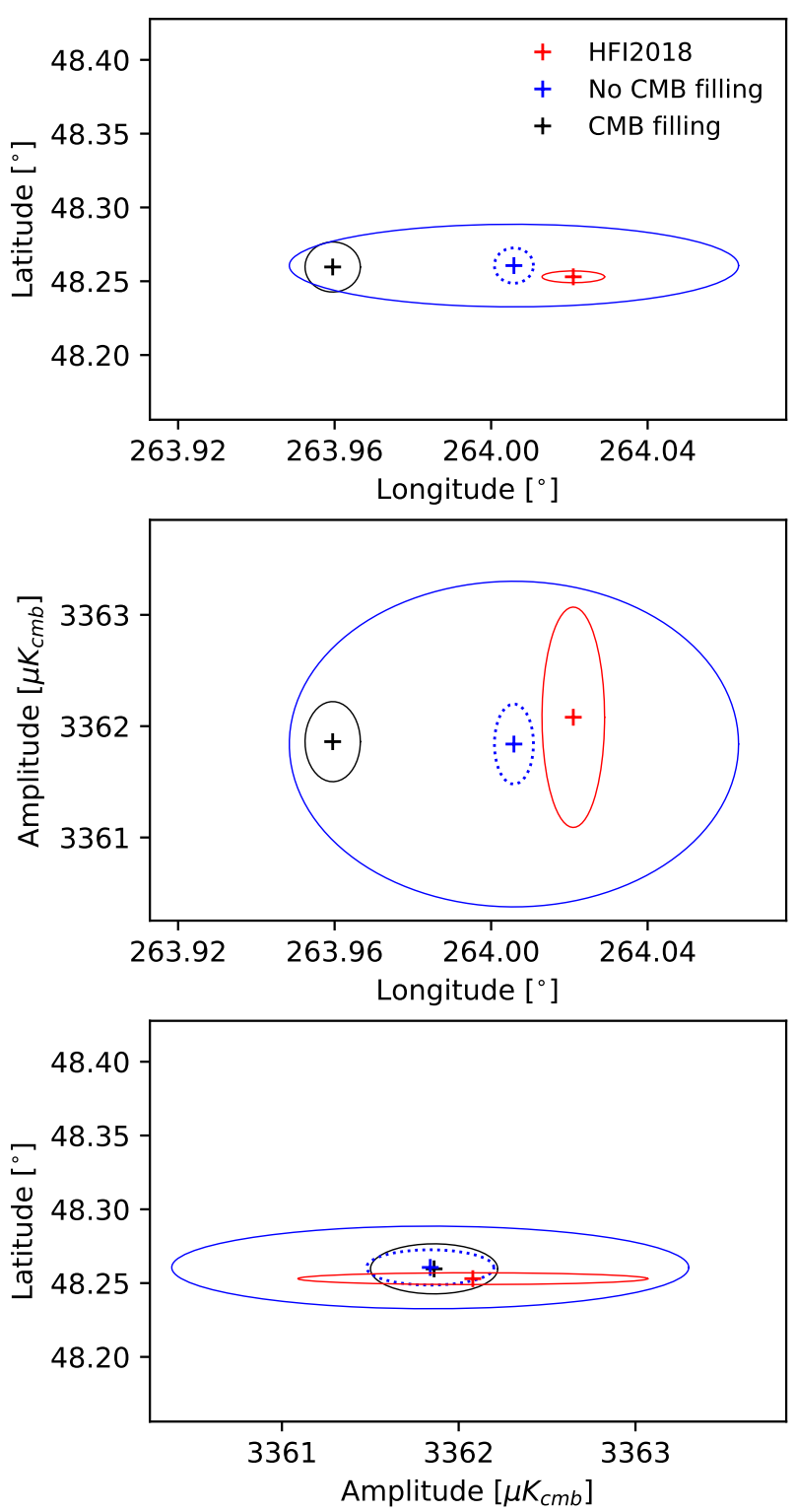

Fig. 11. Three combinations of the solar dipole parameters as a cross together with uncertainty ellipses. Red color shows the HFI-Legacy results. The blue color is the present work but without filling the Galactic ridge mask $M_{\mathrm{g}}$ : dotted when ignoring the systematic effect on the $\mathrm{CMB}$ anisotropies dipolar term and full line when taking into account the systematic effect. The black color is for the results of the present work with Galactic ridge $M_{\mathrm{g}}$ filled with a constrained CMB.

work provides the best measurement of the solar dipole parameters. Nevertheless, the HFI-Legacy systematic uncertainty in longitude is not compatible with the shift of $3.6 \mathrm{arcmin}$ with the new determination in this work. Section 4.4 shows that if the systematic effect associated with the filling of $M_{\mathrm{g}}$ with a CMB realization is included, the compatibility is within $1 \sigma$. This measurement, together with the large-scale CMB cosmological anisotropies map, can be used for cross-calibration of future CMB experiments, and testing the removal of foregrounds as done in this work.

The dust emission is the dominant large-scale anisotropy component above $100 \mathrm{GHz}$ strongly affecting the CMB at the largest angular scales. Such very nonGaussian foreground critically depends on the zero level of the frequency maps which can only be constrained for differential experiments by the coherence 
Table 3. Measurements of the solar dipole parameters for different Collaborations and data sets.

\begin{tabular}{|c|c|c|c|c|}
\hline Reference & Collaboration data set & $\begin{array}{l}\text { Amplitude } \\
{[\mu \mathrm{K}]}\end{array}$ & $\begin{array}{l}\text { Longitude } \\
\text { [deg] }\end{array}$ & $\begin{array}{l}\text { Latitude } \\
\text { [deg] }\end{array}$ \\
\hline Fixsen et al. (1996) & COBE FIRAS & 3372 & $264.14 \pm 0.30$ & $48.26 \pm 0.30$ \\
\hline Hinshaw et al. (2009) & WMAP & 3355 & $263.99 \pm 0.14$ & $48.26 \pm 0.03$ \\
\hline Planck Collaboration I (2016) & Planck HFI+LFI & $3364.5 \pm 2.0$ & $264.00 \pm 0.03$ & $48.24 \pm 0.02$ \\
\hline Planck Collaboration III (2020) & Planck HFI & $\begin{aligned} 3362.08 & \pm 0.09 \text { (stat.) } \\
& \pm 0.45 \text { (syst.) } \\
& \pm 0.45 \text { (cal.) }\end{aligned}$ & $\begin{array}{r}264.021 \pm 0.003 \text { (stat.) } \\
\pm 0.008 \text { (syst.) }\end{array}$ & $\begin{array}{r}48.253 \pm 0.001 \text { (stat.) } \\
\pm 0.004 \text { (syst.) }\end{array}$ \\
\hline This work & Bware HFI & $\begin{array}{r}3361.90 \pm 0.04 \text { (stat.) } \\
\pm 0.36 \text { (syst.) }\end{array}$ & $\begin{array}{r}263.959 \pm 0.002 \text { (stat.) } \\
\pm 0.017 \text { (syst.) }\end{array}$ & $\begin{array}{r}48.260 \pm 0.001 \text { (stat.) } \\
\pm 0.007 \text { (syst.) }\end{array}$ \\
\hline
\end{tabular}

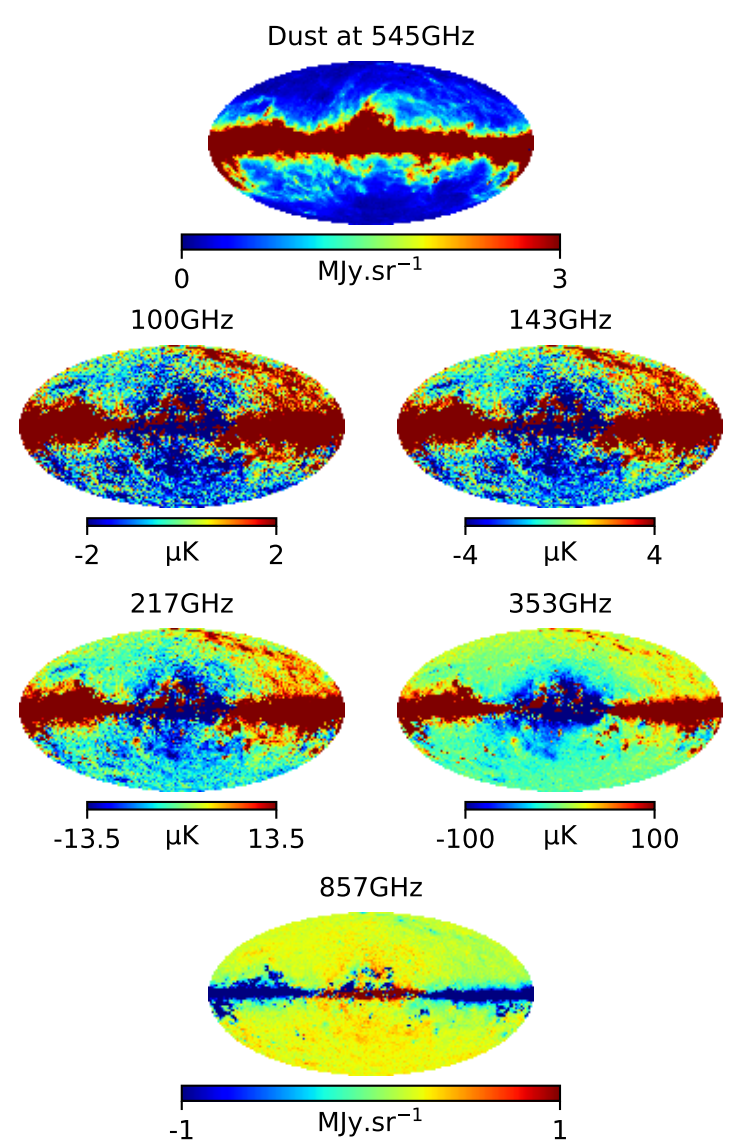

Fig. 12. Dust model maps: the top map shows the Dust template extracted from the $545 \mathrm{GHz}$ final map to be propagated to lower frequencies with the average SED. The other maps show the additive correction accounting for the spatial variation of the dust SED, for each of the other HFI frequencies.

of the dipoles. Here we develop a method that improves these zero levels, which have much better accuracy than the extrapolation to zero column density of interstellar gas. Moreover, the component separation between the $\mathrm{CMB}$ and the dominant dust foreground is performed iteratively and allows intermediate and large-scale maps of the dust SED variations to be built.

The data products associated with this paper are therefore:

- improved zero levels of the HFI single detector maps;

- refined CMB solar dipole vector parameters;
- a new CMB intensity anisotropies map at large scales;

- 100 to $857 \mathrm{GHz}$ dust intensity maps of our new dust model including the SED spatial variation properties.

The intensity final dust model is given in Fig. 12 for each HFI frequency as the sum of the $545 \mathrm{GHz}$ map propagated to other HFI frequencies with the average SED map containing the correction due to the spatially variable dust SED. This correction is not a small one. It will of course affect the dust polarization maps as well and this work is in progress.

The Planck HFI data will remain the best all-sky data at high frequencies for at least a decade. At frequencies higher than $200 \mathrm{GHz}$, the sky signal is strongly affected by the atmosphere. The present study products are therefore unique, and can be used in combination with balloon-borne and ground-based CMB data until the next CMB space mission.

Acknowledgements. This work is part of the Bware project supported by CNES. The authors acknowledge the heritage of the Planck-HFI consortium regarding data, software, knowledge. The program was granted access to the HPC resources of CINES (http://www. cines. fr) under the allocation 2020A0080411364 made by GENCI (http://www.genci.fr). The authors thank Sylvain Mottet for producing the simulations, and Manuel Lopez-Radcenco for improving the wording.

\section{References}

Delouis, J. M., Pagano, L., Mottet, S., Puget, J. L., \& Vibert, L. 2019, A\&A, 629, A38

Fixsen, D. J. 2009, ApJ, 707, 916

Fixsen, D. J., Cheng, E. S., Gales, J. M., et al. 1996, ApJ, 473, 576

Górski, K. M., Hivon, E., Banday, A. J., et al. 2005, ApJ, 622, 759

Hinshaw, G., Weiland, J. L., Hill, R. S., et al. 2009, ApJS, 180, 225

Lagache, G., Abergel, A., Boulanger, F., \& Puget, J. L. 1998, A\&A, 333, 709

Lagache, G., Abergel, A., Boulanger, F., Désert, F. X., \& Puget, J. L. 1999, A\&A, 344,322

Pagano, L., Delouis, J. M., Mottet, S., Puget, J. L., \& Vibert, L. 2020, A\&A, 635, A99

Planck Collaboration VIII. 2014, A\&A, 571, A8

Planck Collaboration IX. 2014, A\&A, 571, A9

Planck Collaboration XI. 2014, A\&A, 571, A11

Planck Collaboration I. 2016, A\&A, 594, A1

Planck Collaboration X. 2016, A\&A, 594, A10

Planck Collaboration III. 2020, A\&A, 641, A3

Planck Collaboration IV. 2020, A\&A, 641, A4

Planck Collaboration V. 2020, A\&A, 641, A5

Planck Collaboration Int. LVII. 2020, A\&A, 643, A42

Planck Collaboration Int. XLVI. 2016, A\&A, 596, A107

Thommesen, H., Andersen, K. J., Aurlien, R., et al. 2020, A\&A, 643, A179

Wandelt, B. D., Larson, D. L., \& Lakshminarayanan, A. 2004, Phys. Rev. D, 70, 083511 


\section{Appendix A: Consistency of the dust model extended to $857 \mathrm{GHz}$}
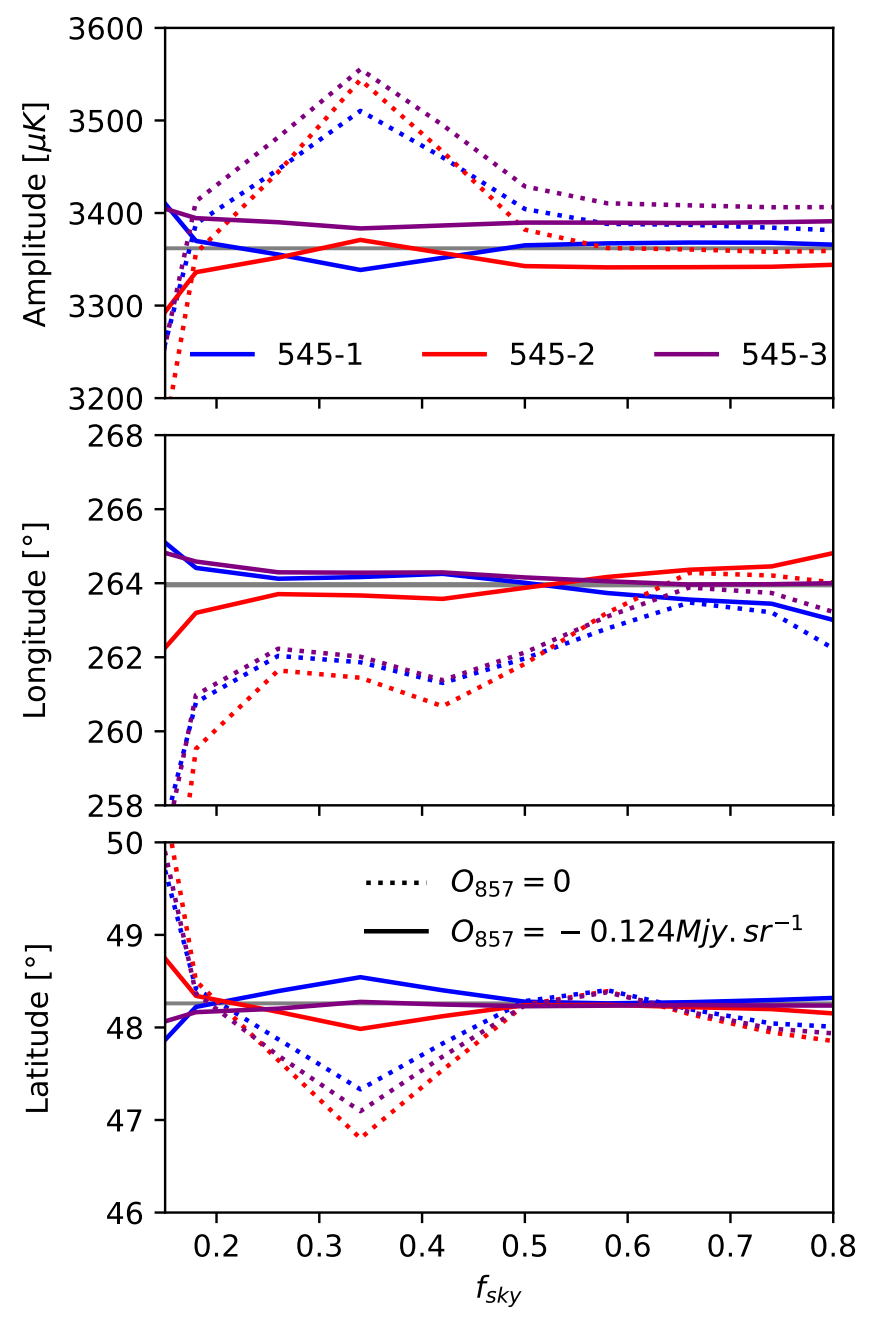

Fig. A.1. Solar dipole parameters extracted from single detector maps at $545 \mathrm{GHz}$ to which a dust map from $857 \mathrm{GHz}$ has been removed. The parameters are shown for the three $545 \mathrm{GHz}$ bolometers, and an offset correction null or optimized to minimize the difference with solar dipole direction.

Figure 2 shows the similarity between the SED correction maps from the HFI-Legacy (based on the $857 \mathrm{GHz}$ map) and the present dust model (based on the $545 \mathrm{GHz}$ ). This suggests that the dust model can be extrapolated to $857 \mathrm{GHz}$.

The $857 \mathrm{GHz}$ map is the sum of the dust emission, an unknown offset correction, noise, and systematic errors. The $\mathrm{CMB}$ anisotropies and low-frequency foreground emission are fully negligible here. Thus, the $857 \mathrm{GHz}$ map is used as a dust template which is propagated at $545 \mathrm{GHz}$ using Eq. (3):

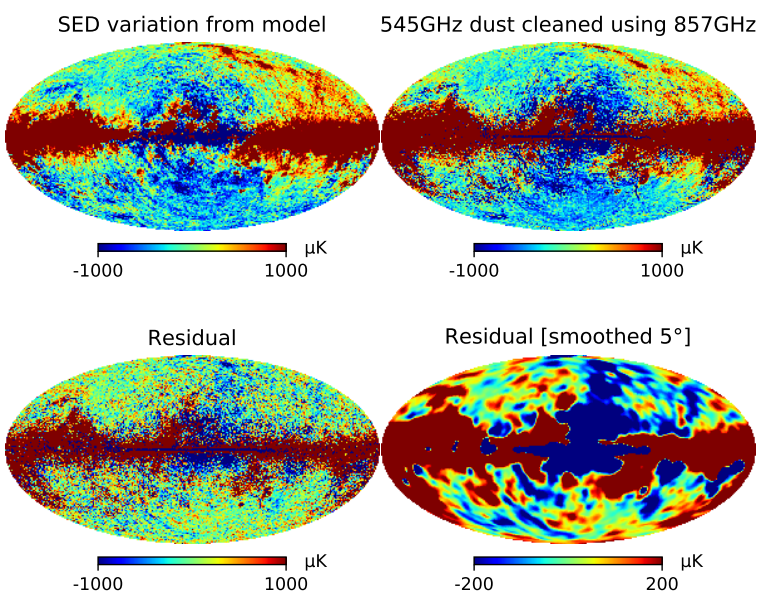

Fig. A.2. Top left map: computed emission associated to the SED dust spatial variation applied to $545 \mathrm{GHz}$. Top right: $545 \mathrm{GHz}$ map correction after linearly subtracting the $857 \mathrm{GHz}$ map. The bottom maps are the differences between the two top maps, smoothed at 1 and $5^{\circ}$. Maps are in $\mu \mathrm{K}_{\mathrm{CMB}}$ to allow comparisons with the dipole amplitude.

$$
\begin{aligned}
\mathcal{D}^{\prime}\left(v_{545}\right)= & \frac{f\left(v_{545}\right)}{f\left(v_{857}\right)} \\
& \times\left[\text { Dust }_{v_{857}}-\ln \left(\frac{v_{857}}{v_{545}}\right) \Delta \text { Dust }_{1}-\ln ^{2}\left(\frac{v_{857}}{v_{545}}\right) \Delta \text { Dust }_{2}\right] .
\end{aligned}
$$

A $545 \mathrm{GHz}$ dust model $\mathcal{D}^{\prime}\left(v_{545}\right)$ is built from the $857 \mathrm{GHz}$ map by propagating the SED variation dust model. This model is removed from the $545 \mathrm{GHz}$ single-detector map, leaving only a CMB solar dipole map plus noise and systematic error residuals. To constrain the $857 \mathrm{GHz}$ map offset $O_{857}$, we compute the solar dipole parameters for each single-detector $545 \mathrm{GHz}$ map following the method described in Sect. 4. The $857 \mathrm{GHz}$ offset is adjusted by minimizing the difference between the $545 \mathrm{GHz}$ solar dipole directions and the 100-217 GHz average (Table 2), and found to be $O_{857}=0.124 \mathrm{MJy} \mathrm{sr}^{-1}$.

Figure A. 1 shows the CMB dipole parameters extracted from the three single-detector $545 \mathrm{GHz}$ maps for both a null $857 \mathrm{GHz}$ offset and for the solved one. The convergence and stability (amplitude dispersion about $50 \mu \mathrm{K}$ ) at such high frequencies demonstrate the quality of the dust model and the fact that the deviations do not come from the dust removal (like in the HFI-Legacy) but more from the photometric calibration errors associated with instrument systematic effects.

Figure A.2 shows the consistency of the two dust maps at $545 \mathrm{GHz}$ (top row). The differences are shown in the bottom row for the full resolution, and at $5^{\circ}$ smoothing. Even if there are some differences near the Galactic ridge, both maps are highly consistent at high latitudes, demonstrating that the dust model stands at large scale and high latitude up to $857 \mathrm{GHz}$. 